This document was prepared in conjunction with work accomplished under Contract No. DE-AC09-96SR18500 with the U. S. Department of Energy.

\title{
DISCLAIMER
}

This report was prepared as an account of work sponsored by an agency of the United States Government. Neither the United States Government nor any agency thereof, nor any of their employees, makes any warranty, express or implied, or assumes any legal liability or responsibility for the accuracy, completeness, or usefulness of any information, apparatus, product or process disclosed, or represents that its use would not infringe privately owned rights. Reference herein to any specific commercial product, process or service by trade name, trademark, manufacturer, or otherwise does not necessarily constitute or imply its endorsement, recommendation, or favoring by the United States Government or any agency thereof. The views and opinions of authors expressed herein do not necessarily state or reflect those of the United States Government or any agency thereof.

This report has been reproduced directly from the best available copy.

Available for sale to the public, in paper, from: U.S. Department of Commerce, National Technical Information Service, 5285 Port Royal Road, Springfield, VA 22161, phone: (800) 553-6847, fax: (703) 605-6900

email: orders@ntis.fedworld.gov

online ordering: http://www.ntis.gov/help/index.asp

Available electronically at http://www.osti.gov/bridge

Available for a processing fee to U.S. Department of Energy and its contractors, in paper, from: U.S. Department of Energy, Office of Scientific and Technical Information, P.O. Box 62, Oak Ridge, TN 37831-0062,

phone: (865)576-8401,

fax: (865)576-5728

email: $\underline{\text { reports@ adonis.osti.gov }}$ 
WSRC-TR-2001-00584, Revision 0

SRT-RPP-2001-00220, Revision 0

\section{Results from Analysis of a Hanford Tank 241-AN-102 Melter-Scale Glass Waste Form}

Westinghouse Savannah River Company

Savannah River Site

Aiken, SC 29808

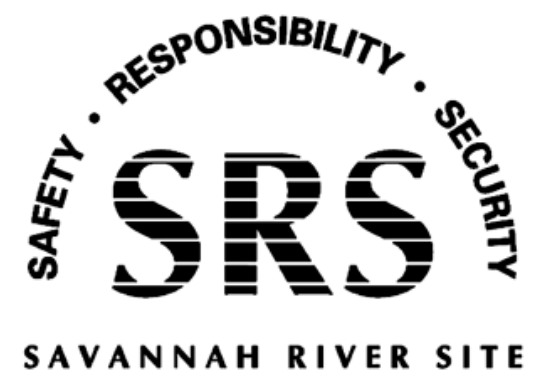

Prepared for the U.S. Department of Energy under Contract No. DE-AC09-96SR18500 
WSRC-TR-2001-00584, Revision 0

SRT-RPP-2001-00220, Revision 0

KEYWORDS:

Hanford River Protection Project, Vitrification, Low-Activity Waste,

Envelope C, AN-102, Regulatory

\section{Results from Analysis of a Hanford Tank 241-AN-102 Melter-Scale Glass Waste Form}

D. M. Ferrara

R. J. Ray

C.s L. Crawford

W. P. Kubilius

R. F. Schumacher

Publication Date: April 26, 2002

Westinghouse Savannah River Company

Savannah River Site

Aiken, SC 29808

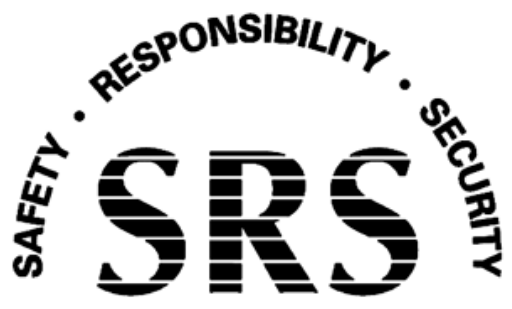

SAVANNAH RIVER SITE

Prepared for the U.S. Department of Energy under Contract No. DE-AC09-96SR18500 


\section{Acknowledgements}

The results presented in this report are the product of efforts from more than twenty individuals at the Savannah River Site and at Babcock and Wilcox Technology (BWXT) Services, Inc. The success of this program was only possible because of the ability of these individuals to work as a team. In particular, the authors would like to thank personnel at BWXT Services, Inc. Our primary contacts were Jim Clark, Paul Macek and Les Hawthorne at BWXT Services, Inc. In addition, at the Savannah River Site, we are thankful for the extra effort that was put into the sample preparation and shipment by Susan Jurgensen, Sarah Brown, Sherry Vissage, and Beverly Burch. Contributions from Steve Martin and John Connelly were also essential in our ability to establish this program and to meet our customer's quality requirements.

\section{Contents}

Tables $v$

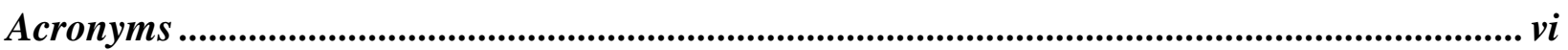

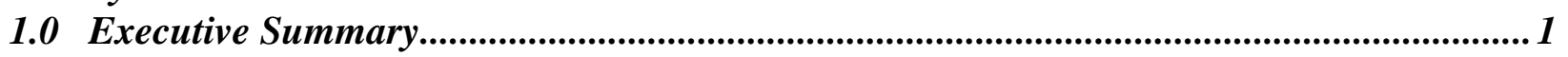

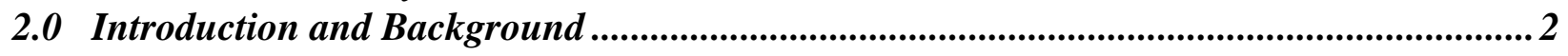

3.0 Experimental ...................................................................................................................... 3

3.A Target Organic Analytes, TCLP, and Physical Properties .............................................................. 3

3.B Target Inorganic Analytes and Radionuclides ......................................................................

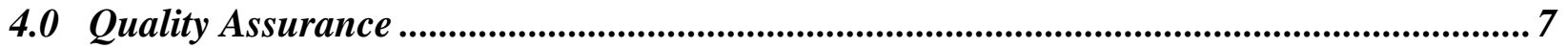

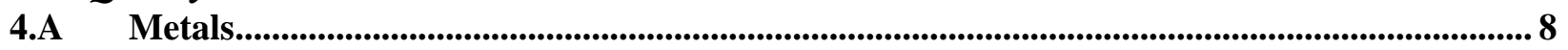

4.B Inorganic Analytes Detected as Ionic Species ...................................................................................... 14

4.C Radionuclide Analyses ........................................................................................................................ 14

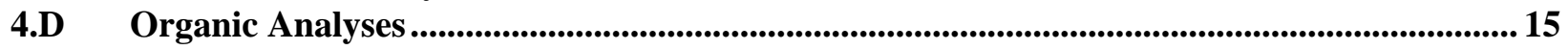

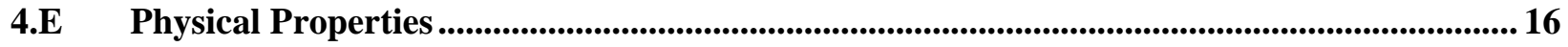

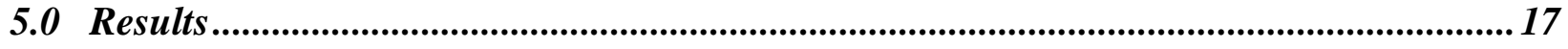

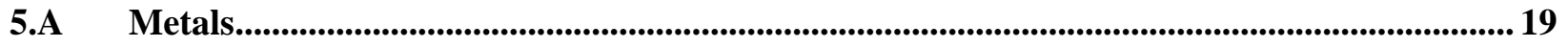

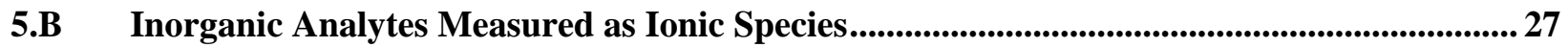

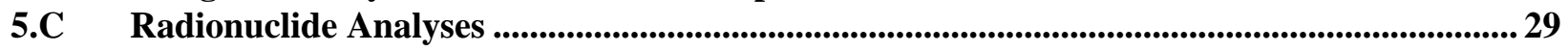

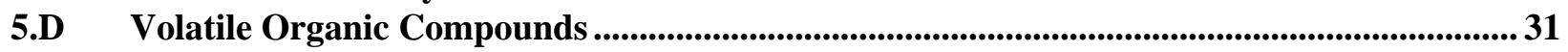

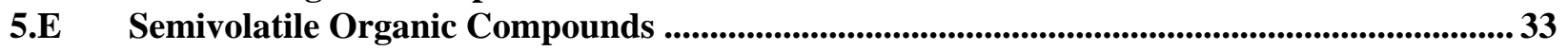

5.F Pesticides and Polychlorinated Biphenyls (PCBs) ............................................................................. 35

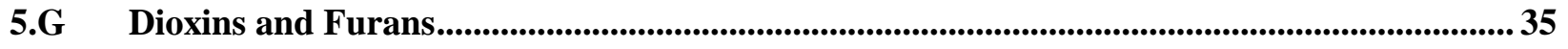

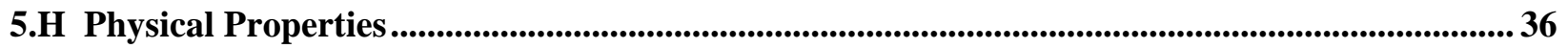

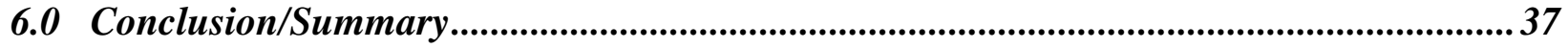

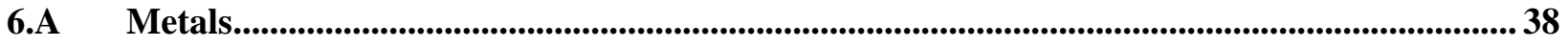

6.B Inorganic Analytes Measured as Ionic Species................................................................................ 39

6.C Radionuclides ................................................................................................................................................39

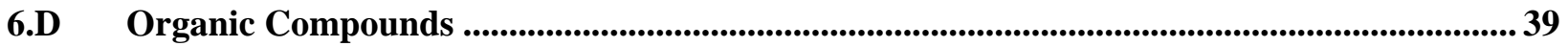

6.E Physical Properties ............................................................................................................................................ 40 


\section{Tables}

Table 3.1. Methods Used to Prepare and to Analyze Undissolved Glass Samples .......................................... 5

Table 3.2. Methods Used to Prepare and to Analyze Dissolved Glass Samples........................................................ 6

Table 4.1. Summary of Percent Completeness ..................................................................................................9

Table 4.2. Recoveries and RPD Values for Non-RCRA Metals ................................................................... 10

Table 4.3. Detection and Quantitation Limits for Non-RCRA Metals ............................................................... 11

Table 4.4. Recoveries for RCRA Metals .......................................................................................................... 12

Table 4.5. Detection and Quantitation Limits for RCRA Metals ................................................................ 13

Table 4.6 Recoveries, and Detection and Quantitation Limits for the TCLP Extract Analyses ........................ 13

Table 4.7. Method 9056 Recovery, Reproducibility, and Detection and Quantitation Limits .......................... 14

Table 4.8. Results from Method 9056 Analyses of Blanks and Standard.......................................................... 15

Table 4.8. Minimum Detectable Activities ....................................................................................................... 16

Table 4.9. Recoveries and Quantitation Limits for Volatile Organic Analyses .............................................. 17

Table 4.10. Recoveries and Quantitation Limits for Miscellaneous Organic Analyses ...................................... 18

Table 5.1. Concentrations of Target Non-RCRA Metal Analytes Measured by ICP-ES and AA .................. 20

Table 5.2. Concentrations of RCRA Metal Analytes Measured by ICP-ES and AA...................................... 21

Table 5.3. Non-RCRA Metal Results Compared to SRTC Results and to the Target Composition ................ 23

Table 5.4. RCRA Metal Results Compared to SRTC Results and to the Target Composition.......................... 24

Table 5.5. Non-RCRA Metal Results Compared to Results from a Crucible-Scale Vitrification..................... 25

Table 5.6. RCRA Metal Results Compared to Results from a Crucible-Scale Vitrification .............................. 26

Table 5.7. Concentration of RCRA Metals in TCLP Extract .................................................................................. 27

Table 5.8. Concentration of Target Inorganic Analytes Measured as Ionic Species......................................... 28

Table 5.9. Comparison of Target Inorganic Analytes Measured as Ionic Species .............................................. 28

Table 5.10. Concentration of Target Radionuclides ...................................................................................................... 30

Table 5.11. Comparison of Target Radionuclides ............................................................................................................ 32

Table 5.12. Concentrations of Target Volatile Organic Analytes........................................................................ 33

Table 5.14. Concentrations of Pesticides and Polychlorinated Biphenyls.......................................................... 36

Table 5.15. Concentrations of Dioxins and Furans ......................................................................................... 37 


\section{Acronyms}

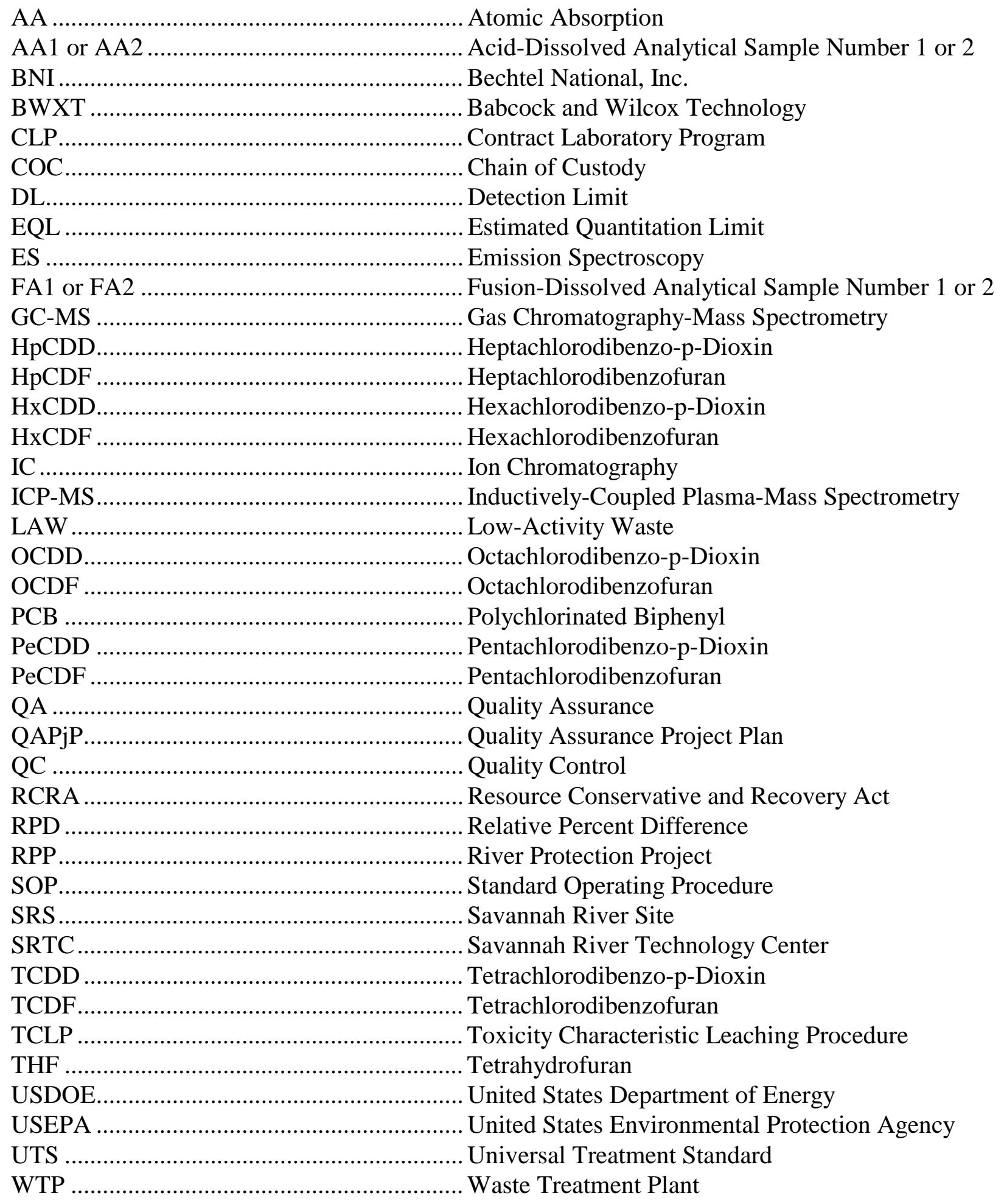




\subsection{Executive Summary}

Analyses were performed to characterize a low-activity glass waste form that was produced from a research-scale melter using a Hanford Tank AN-102 supernate that had been treated to remove strontium, transuranic elements, cesium, and technetium. One of the goals of the analyses was to perform the characterization using protocol that would produce results in a form compatible with regulatory applications. As a result, the laboratory chosen to perform these analyses was one that was capable of meeting USEPA protocol. These analyses showed

-The measured waste form composition was consistent with the target composition (provided by the Vitreous State Laboratory) for primary components (metals, sulfur, phosphorous, and halides).

-The waste form met requirements of the Washington Administrative Code (WAC).

-No organic species were detectable in the waste form.

-Results from analyses performed according to USEPA protocol were similar to results from analyses performed at SRTC.

-The composition of the waste form was similar to that for crucible-scale studies except for technetium.

For elements present in the glass waste form at concentrations of $10^{6} \mu \mathrm{g} / \mathrm{kg}$ or higher $(1000 \mathrm{ppm}$ or higher), the waste form composition was similar to the target composition. On an oxide basis, elements that were present above the estimated quantitation limits (EQLs) accounted for $100 \%$ of the waste form composition after adjusting for titanium (which was not a target analyte). Of the elements present above the EQLs, only the measured manganese concentration deviated from the target composition by more than $20 \%$.

The waste form met limits set by the WAC for radionuclides present, for release of toxic metals, and for flammability. Strontium-90, cesium-137, technetium-99, and alpha-emitting transuranic radionuclide concentrations were below limits set on these radionuclides. In addition, Toxicity Characteristic Leaching Procedure results were below maximum allowable concentrations for all metals that were measured (vanadium and zinc were not measured). Of course, the waste form was not ignitable at temperatures up to $1200{ }^{\circ} \mathrm{C}$. The waste form therefore did not demonstrate the characteristic of ignitability, because it was not a) a liquid, b) an ignitable compressed gas, c) an oxidizer, and d) capable of causing a fire under standard temperature and pressure.

As expected, no organic compounds were detected. Measurements were made for volatile organic compounds, semivolatile organic compounds, pesticides and PCBs, and dioxins and furans. Although five compounds were detected during semivolatile organic analyses and ten of the dioxins and furans were detected at low concentrations, these analytes were also detected in the associated field, trip, and method blanks at similar or higher concentrations. 


\section{WSRC-TR-2001-00584, Revision 0 \\ SRT-RPP-2001-00220, Revision 0}

Results were compared to SRTC analyses reported previously for this melter glass waste form and were compared to analyses performed on a Tank AN-102 glass waste form produced on a crucible scale. Although minor differences were observed between the results determined by the subcontract laboratory and the SRTC results, these differences were rare. In addition, the waste form produced from the melter demonstration was similar to the crucible-scale waste form except for technetium. More technetium appeared to be released to the off gas during melter tests.

\subsection{Introduction and Background}

Bechtel National, Inc. (BNI) has been awarded a contract from the United States Department of Energy's (USDOE's) Office of River Protection to develop, design, construct, and startup the River Protection Project Waste Treatment Plant (RPP-WTP). The RPP-WTP will pretreat and immobilize the radioactive waste that is being stored in underground storage tanks at the USDOE Hanford Site outside Richland, Washington.

As part of the RPP-WTP, BNI and USDOE have contracted Westinghouse Savannah River Company's Savannah River Technology Center (SRTC) to perform process demonstrations using nonradioactive samples (waste stream simulants) and radioactive samples (from the Hanford Tank Farm). The work being performed (including the task described in this report) has been performed by SRTC under the technical direction of BNI. The specifications under which regulatory analyses have been performed were communicated to SRTC in a project quality assurance project plan (QAPjP). ${ }^{1}$

One radioactive sample that was shipped to SRTC for pretreatment and vitrification process demonstration was a thirty-liter supernate sample from Hanford Tank 241-AN-102. The "241" is used to designate all Hanford radioactive underground waste storage tanks. Throughout the rest of this report the sample will be referred to as "AN-102". Upon receipt at SRTC, the sample was characterized and pretreated to remove transuranic elements and strontium, cesium, and technetium.

A small sample of the resulting low-activity waste supernate was mixed with glass-forming minerals and vitrified in a crucible. This glass waste form was crushed, sampled, prepared, and sent to Babcock and Wilcox Technology (BWXT) Services, Inc. for analysis. Details of the vitrification and results from analysis of this crucible-scale glass waste form have been reported elsewhere. $^{2,3}$

\footnotetext{
${ }^{1}$ Jennifer Su-Coker, "Quality Assurance Project Plan for Testing Programs: Savannah River Technology Center (SRTC), Pacific Northwest National Laboratory (PNNL),” QP-W375-EN00002, Rev. 0, June 7, 1999.

${ }^{2}$ C. L. Crawford, D. M. Ferrara, R. F. Schumacher, N. E. Bibler, "Crucible-Scale Active Vitrification Testing Envelope C, Tank 241-AN-102 (U)," SRT-RPP-2000-00022, Rev. 0, June 15, 2001.
} 


\section{WSRC-TR-2001-00584, Revision 0 \\ SRT-RPP-2001-00220, Revision 0}

After the aliquot had been removed for the crucible-scale vitrification, the rest of the sample was concentrated in a bench-scale evaporator and then fed to a bench-scale melter producing a glass waste form that was rapidly cooled. To better replicate conditions under which the waste form would be produced, the resulting glass was then transferred to a furnace, brought back to melt temperature $\left(1150{ }^{\circ} \mathrm{C}\right)$ and cooled following a more prototypic temperature schedule. Details of the evaporator and melter operations have been presented elsewhere. ${ }^{4}$

This report is a summary of results from characterization of the glass waste form that was produced from pretreatment and melter vitrification of this supernate sample. The objectives of these analyses were to

-characterize the waste form using protocol that allows for regulatory applications of the results -determine whether the waste form met specifications of the Washington Administrative Code -compare results to values from analysis of the crucible-scale glass waste form -compare results to values determined at SRTC using standard SRTC characterization methods

\subsection{Experimental}

The melter study was performed in three stages, two tests with nonradioactive Tank AN-102 simulants and one with the treated Tank AN-102 sample. Details of these tests have been given elsewhere. ${ }^{4}$ The actual Tank AN-102 glass waste form was sampled, prepared, and analyzed according to USEPA SW-846 protocol. In addition, samples were taken of glass from one of the nonradioactive tests. This nonradioactive material was analyzed as a vitrification "process blank." The general approach to the sample preparation and analysis has been given in Figure 1.

\section{A Target Organic Analytes, TCLP, and Physical Properties}

After vitrification, glass monoliths were crushed into particles with diameters of less than 0.9 centimeter. This initial size reduction was performed with an agate mortar and pestle taking precautions to eliminate contact with organic material. ${ }^{4}$ In addition to the Tank AN-102 samples and process blanks, a low-activity test reference material ${ }^{5}$ was crushed to generate field blanks. Although the low-activity test reference material glass contained some of the target analytes, it was the only option available since material typically used for blanks (e.g. water) would not have been a reasonable matrix for steps such as size reduction.

${ }^{3}$ D. M. Ferrara, R. J. Ray, C. L. Crawford, and Walter P. Kubilius, "Results from Analyses of a Hanford Tank AN102 Crucible-Scale Glass Waste Form,” SRT-RPP-2001-00013, Rev. 1, November 16, 2001.

${ }^{4}$ J. R. Zamecnik, C. L. Crawford, and D. C. Koopman, "Task Technical and QA Plan: Large Scale Vitrification of 241-AN-102 (Envelope C) Sample,” SRT-RPP-2001-00034, Rev. 0, April 2002.

${ }^{5}$ W. L. Ebert and S. F. Wolf, “An Interlaboratory Study of a Standard Glass for Acceptance Testing of Low-Activity Waste Glass,” Journal of Nuclear Materials, 282, 2000, pg. 112-124. 


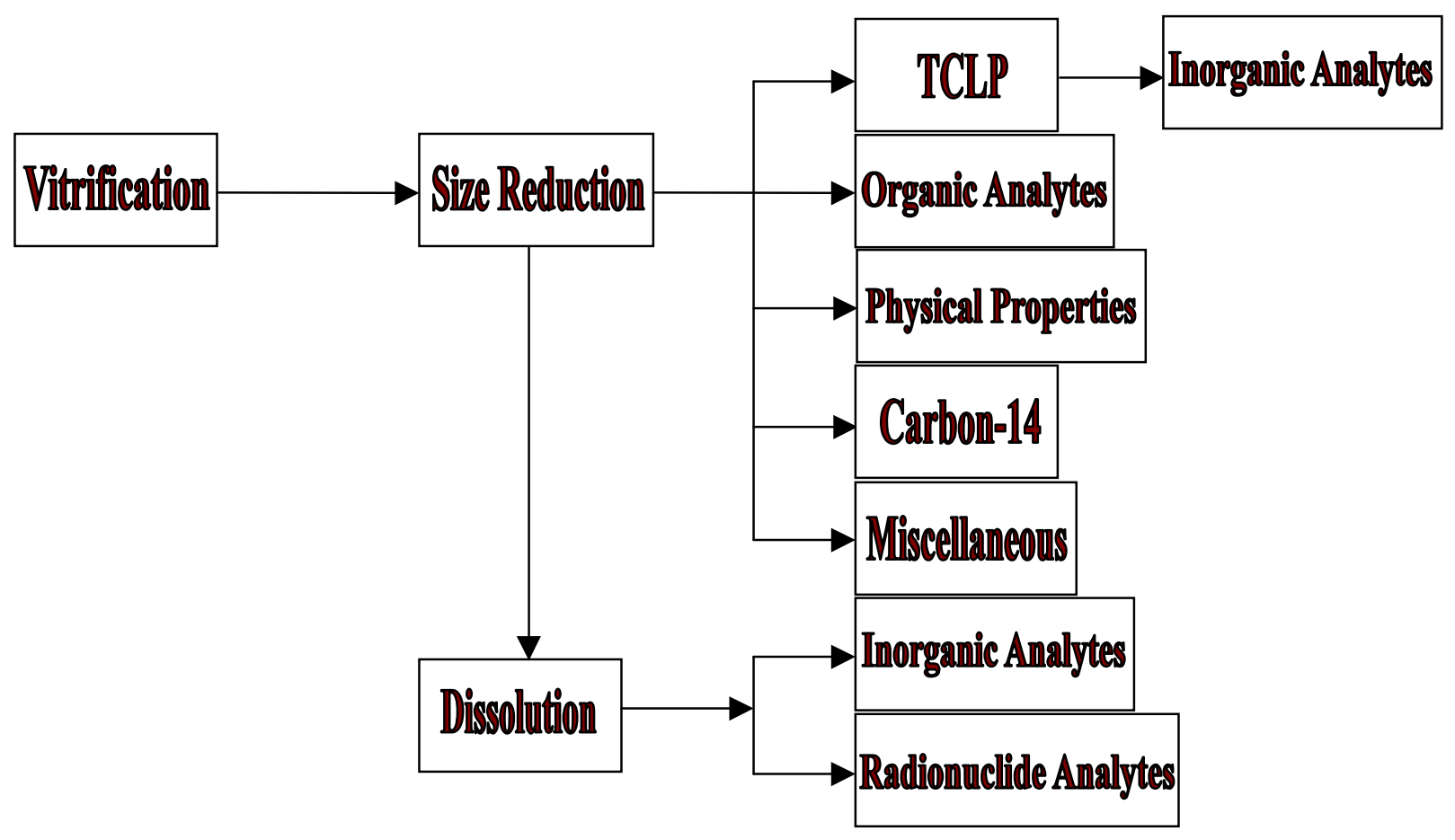

Figure 1. Flowchart for Pretreatment and Analysis of the Tank AN-102 Glass Waste Form

Upon completion of the initial size reduction, samples were collected and shipped to Babcock and Wilcox Technology (BWXT) Services for further preparation and analysis as shown in Table 3.1. As indicated, most of these sample preparations and analyses were performed by a straightforward application of the USEPA SW-846 methods. Methanol and 2-butoxyethanol were extracted using USEPA SW-846 Method 5035, modified to use water as the extraction fluid.

Total halide and carbon-14 analyses were to be performed on the undissolved samples as well as the dissolved samples because each approach has limitations. Dissolution of the glass samples can cause loses of these analytes. In addition, hydrofluoric acid (which was used in one of the three dissolution methods employed) makes total halide determinations impossible in the aciddissolved samples. Analyses of the undissolved samples are also limited. USEPA SW-846 Method 9020 specifically states that it does not measure halide in undissolved species.

\section{B Target Inorganic Analytes and Radionuclides}

As shown in Figure 1, most of the inorganic and radionuclide analytes were determined in samples that were dissolved. The dissolved glass samples were shipped to BWXT Services for analysis. The analytical methods used for these analyses have been given in Table 3.2. As indicated in the previous section, some of the inorganic analytes and carbon-14 were determined in both the dissolved samples and in undissolved samples. 
WSRC-TR-2001-00584, Revision 0

SRT-RPP-2001-00220, Revision 0

Table 3.1. Methods Used to Prepare and to Analyze Undissolved Glass Samples

\begin{tabular}{|c|c|c|c|}
\hline Analytes & Sample ID & Preparation & Analysis \\
\hline Volatile Organic Compounds & VA1, VA2 & SW-846 \# 5035 & SW-846 \# 8260B \\
\hline Methanol, 2-Butoxyethanol & IA1, IA2 & * SW-846 \# 5035 & SW-846 \# 8260B \\
\hline Semivolatiles Organic Compounds & SA1, SA2 & SW-846 \# 3540 & SW-846 \# 8270C \\
\hline Triethylamine & SA1, SA2 & SW-846 \# 3540 & ICP-MS \\
\hline Pesticides & PA1, PA2 & SW-846 \# 3540 & SW-846 \# 8081A \\
\hline Polychlorinated Biphenyls & PA1, PA2 & SW-846 \# 3540 & SW-846 \# 8081A \\
\hline Dioxins and Furans & DA1, DA2 & - & SW-846 \# 7470A \\
\hline Organic Anions & IA1, IA2 & Hot Water Leach & SW-846 \# 9056 \\
\hline Miscellaneous RCRA Metals & TA1 & SW-846 \# 1311, 3015 & SW-846 \# 6010B \\
\hline Arsenic & TA1 & SW-846 \# 1311, 3015 & SW-846 \# 7060A \\
\hline Mercury & TA1 & SW-846\# 1311, 3015 & SW-846 \# 7470A \\
\hline Selenium & TA1 & SW-846 \# 1311, 3015 & SW-846 \# 7740 \\
\hline Ammonia & BA1, BA2 & Digestion & Potentiometry \\
\hline Cyanide & BA1, BA2 & SW-846 \# 9010B & SW-846 \# 9014 \\
\hline Total Halides & BA1, BA2 & - & **SW-846 \# 9020 \\
\hline Ignitability & BA1, BA2 & - & Mod. SW-846 \#1010 \\
\hline Carbon-14 & BA1, BA2 & Oxidation & Liquid Scintillation \\
\hline
\end{tabular}

*USEPA SW-846 Method 5035 was modified to use a water extraction fluid for these samples. **USEPA SW-846 Method 1010 was modified to use smaller samples than specified.

\section{B.1 Sample Dissolution}

After the Tank AN-102 waste form, the process blanks, and the field blank were subjected to the initial size reduction steps, a portion of the resulting less than $0.9 \mathrm{~cm}$ material was ground further using a Mixer Mill with agate cups and an agate grinding ball. ${ }^{4}$ The resulting material was verified to be ground to less than 200 mesh by passing the material through an ASTM-certified brass sieve.

Aliquots of this finely-ground material were then dissolved using three different dissolution techniques. ${ }^{4}$ Each of these dissolutions resulted in two AN-102 samples, two process blanks, two standards (dissolved low-activity test reference material glass), a field blank, and a method blank. Dissolution methods were

-dissolution with hydrofluoric, nitric, and boric acid heated to $105^{\circ} \mathrm{C}$ in a closed plastic bottle -dissolution using a sodium peroxide fusion method followed by a nitric acid uptake -dissolution using a sodium peroxide fusion method followed by a water uptake 
The dissolved samples were then shipped to BWXT where they were prepared and analyzed. The methods used to prepare and analyze these samples have been given in Table 3.2. Where possible, standard USEPA SW-846 methods were used to perform these preparations and analyses. At all stages in the sampling, handling, shipping, preparation, and analysis, USEPA SW-846 protocol was followed except where noted in this report.

Table 3.2. Methods Used to Prepare and to Analyze Dissolved Glass Samples

\begin{tabular}{|c|c|c|c|c|}
\hline Analytes & Sample ID & Dissolution & Preparation & Analysis \\
\hline \multirow{2}{*}{$\begin{array}{c}\text { Miscellaneous } \\
\text { Metals }\end{array}$} & $\mathrm{AA} 1, \mathrm{AA} 2$ & Acid & \multirow{2}{*}{$\begin{array}{c}\text { SW-846 } \\
\# 3015 \\
\end{array}$} & \multirow{2}{*}{$\begin{array}{l}\text { SW-846 } \\
\# 6010 B \\
\end{array}$} \\
\hline & FA1, FA2 & Fusion with Acid Uptake & & \\
\hline \multirow{2}{*}{ Arsenic } & AA1, AA2 & Acid & \multirow{2}{*}{$\begin{array}{c}\text { SW-846 } \\
\# 3015\end{array}$} & \multirow{2}{*}{$\begin{array}{l}\text { SW-846 } \\
\# \text { 7060A }\end{array}$} \\
\hline & FA1, FA2 & Fusion with Acid Uptake & & \\
\hline \multirow{2}{*}{ Selenium } & AA1, AA2 & Acid & \multirow{2}{*}{$\begin{array}{c}\text { SW-846 } \\
\# 3015 \\
\end{array}$} & \multirow{2}{*}{$\begin{array}{c}\text { SW-846 } \\
\# 7740 \\
\end{array}$} \\
\hline & FA1, FA2 & Fusion with Acid Uptake & & \\
\hline Mercury & $\mathrm{AA} 1, \mathrm{AA} 2$ & Acid & - & SW-846 \# 7470A \\
\hline \multirow{2}{*}{$\begin{array}{c}\text { Gross Alpha } \\
\text { and Beta } \\
\end{array}$} & $\mathrm{AA} 1, \mathrm{AA} 2$ & Acid & \multirow{2}{*}{ - } & \multirow{2}{*}{$\begin{array}{l}\text { SW-846 } \\
\# 9310 A\end{array}$} \\
\hline & FA1, FA2 & Fusion with Acid Uptake & & \\
\hline \multirow{2}{*}{$\begin{array}{c}\text { Alpha-Emitting } \\
\text { Radionuclides }\end{array}$} & AA1, AA2 & Acid & \multirow{2}{*}{ Separation } & \multirow{2}{*}{$\begin{array}{c}\text { Alpha } \\
\text { Spectroscopy }\end{array}$} \\
\hline & FA1, FA2 & Fusion with Acid Uptake & & \\
\hline \multirow{2}{*}{$\begin{array}{c}\text { Pure Beta-Emitting } \\
\text { Radionuclides }\end{array}$} & AA1, AA2 & Acid & \multirow{2}{*}{ Separation } & \multirow{2}{*}{$\begin{array}{c}\text { Liquid } \\
\text { Scintillation }\end{array}$} \\
\hline & FA1, FA2 & Fusion with Acid Uptake & & \\
\hline \multirow{2}{*}{$\begin{array}{c}\text { Gamma-Emitting } \\
\text { Radionuclides }\end{array}$} & $\mathrm{AA} 1, \mathrm{AA} 2$ & Acid & & \multirow{2}{*}{$\begin{array}{c}\text { Gamma } \\
\text { Spectroscopy }\end{array}$} \\
\hline & FA1, FA2 & Fusion with Acid Uptake & & \\
\hline \multirow{2}{*}{ Iodine } & AA1, AA2 & Acid & & \multirow[t]{2}{*}{ Colorimetry } \\
\hline & FA1, FA2 & Fusion with Acid Uptake & & \\
\hline Inorganic Anions & WA1,WA2 & Fusion with Water Uptake & Na removal & SW-846 \# 9056 \\
\hline
\end{tabular}

\section{B.2 Sample Analysis}

Most metals were determined by USEPA SW-846 Method 6010B which is an ICP-ES procedure. Because of interferences associated with the dissolution processes, sodium, potassium, and nickel were not reported in the peroxide fusion samples and boron was not reported in the aciddissolved samples. In addition to the $200 \mathrm{~mL} / \mathrm{g}$ "dilution" that occurred during the dissolution, samples were further diluted by a factor of 20 or 100 during the USEPA SW-846 Method 3015 sample preparation and subsequent analysis. For boron and silicon, this factor was 200. 


\section{WSRC-TR-2001-00584, Revision 0 \\ SRT-RPP-2001-00220, Revision 0}

Arsenic and selenium were determined by atomic absorption (USEPA SW-846 Methods 7060A and 7740, respectively) in addition to being determined during the Method 6010B analyses. Prior to analysis, acid dissolved samples were diluted by a factor of 20 and fusion samples were diluted by a factor of 100. Mercury was determined by Method 7470A. Mercury was not determined in the peroxide fusion samples because the peroxide fusion was performed at elevated temperatures in an open vessel. Most of the mercury would have been lost from the sample under these conditions. Samples were not diluted prior to the mercury analysis.

Radionuclides were determined as indicated in Table 3.2. Alpha spectroscopy was used for neptunium-237, americium-241, and uranium, curium and plutonium isotope analyses (except plutonium-241). Liquid scintillation was used to measure tritium, carbon-14, selenium-79, strontium-90, technetium-99, and plutonium-241. Gamma spectroscopy was used for cobalt-60, niobium-94, radium-226, and ruthenium, europium and cesium isotope analyses. Americium241 results from gamma spectroscopy were reported as well as the alpha spectroscopy results.

Because of anions introduced during the dissolution process, inorganic anions could only be determined on samples that were dissolved by peroxide fusion followed by a water uptake. In such samples, the high sodium concentrations interfere with the chloride and fluoride determinations. ${ }^{3}$ Therefore, prior to performing the USEPA SW-846 Method 9056 analyses, the sodium hydroxide interference was removed from the samples using Dionex OnGuard ${ }^{\oplus}$ II H, 1-cc cartridges. These cartridges use a styrene-based sulfonic acid resin to remove sodium, alkaline earth metals, and transition metals.

Iodine was measured in the acid-dissolved samples and in the peroxide samples. The total halide was to be measured in these samples by USEPA SW-846 Method 9020B; however, the laboratory's total halide analyzer was out of service. An upper limit for the total halide can be determined by adding the bromide, chloride and fluoride results from USEPA SW-846 Method 9056 to the results from the iodine colorimetry.

\subsection{Quality Assurance}

As indicated previously, samples were collected, prepared, and analyzed according to USEPA SW-846 protocol to the greatest extent practical, even for target analytes not addressed in USEPA SW-846 (e.g. for radionuclides). Results were validated according to the general criteria given for "Definitive Data" in "Data Quality Objectives for Superfund," Interim Final, EPA540R-93-071, September 1993 and "EPA National Functional Guidelines for Organic Data Review," Revised Draft, June 1991. Aspects reviewed during the validation were

-Chain of Custody (COC): COC documents completed correctly and signed

-Sample Documentation: All relevant documentation completed and included in data package

-Sample Preservation: Verification that samples were held at $4{ }^{\circ} \mathrm{C}$ when appropriate

-Hold Times: Samples prepared and analyzed according to hold time for analytes of interest -Initial and Continuing Calibration: Initial and continuing calibration within appropriate range -Analyte Identification: Analyte correctly identified (e.g. mass spectrometry tune validation) 


\section{WSRC-TR-2001-00584, Revision 0 \\ SRT-RPP-2001-00220, Revision 0}

-Analyte Quantitation: Concentration correctly determined (e.g. lack of interferences)

-Method Blanks: Method blank concentrations sufficiently below sample concentrations

-Matrix Spike Recoveries: Recovery within specified range and recovery precision as specified

-Surrogate, Tracer, and Carrier Recoveries: Recovery within range specified for matrix spike

-Laboratory Control Sample Recoveries: Recovery within range specified for matrix spike

-Laboratory Duplicates: Precision within range allowed for by specific USEPA SW-846 method

-Trip Blanks (where appropriate): Concentrations sufficiently below sample concentrations

Matrix spike and matrix spike duplicate results have been used to determine accuracy as measured by recoveries and precision as measured by relative percent difference (RPD). Both have been calculated according to USEPA SW-846. Recovery has been calculated from the concentration due to the spike (total measured concentration minus unspiked sample concentration). As specified in USEPA SW-846, RPD has been determined using the total measured concentrations of the matrix spike and matrix spike duplicate (not from the concentration due to the spike alone).

Analytes were diluted during most of the sample preparation steps. Detection limits (DLs) and EQLs have been designated as "method" or "sample" limits. In this report, the difference between method limits and sample limits is the factor by which the analytes were diluted prior to analysis. Method DLs and EQLs reflect minimum concentrations measurable in the samples after they have been digested, diluted etc. Sample DLs and EQLS are the corresponding minimum concentrations in the initial glass waste form sample.

Of 524 sample measurements, 460 were determined to be valid and 64 to be invalid. Thirtyseven results were considered invalid because recoveries were outside specified limits, fourteen because RPDs were too high, four because of interferences, seven because absence of a key peak, and two density measurements because of poor precision. Although a minimum completeness requirement was not specified for this task, the corresponding percent completeness $(88 \%)$ was slightly lower than the minimum that will be imposed on similar tasks $(90 \%)$. These results have been summarized in Table 4.1.

\section{A Metals}

Elemental analysis of the Tank AN-102 glass waste form has been presented in this report as three groups, non-RCRA metals, RCRA metals, and TCLP results. The non-RCRA metals and RCRA metals are being presented separately to simplify use of the information for personnel interested primarily in the RCRA metalconcentrations. This section is a presentation of the QA and QC results for the elemental analyses. 
WSRC-TR-2001-00584, Revision 0

SRT-RPP-2001-00220, Revision 0

Table 4.1. Summary of Percent Completeness

\begin{tabular}{|c|c|c|c|c|c|}
\hline \multicolumn{1}{|c|}{ Analyses } & \multicolumn{3}{|c|}{ Results } & Completeness & Cause of Invalid Results \\
\hline & Total & Valid & Invalid & $(\%)$ & \\
\hline Non-RCRA Metals & 100 & 88 & 12 & 88 & Unacceptable Recoveries \\
\hline RCRA Metals & 60 & 56 & 4 & 93 & Unacceptable Recoveries \\
\hline TCLP & 14 & 13 & 1 & 93 & Unacceptable Recovery \\
\hline Ions & 20 & 14 & 6 & 70 & Unacceptable Recoveries \\
\hline Radionuclides & 120 & 109 & 11 & 91 & Interferences, No peak \\
\hline Volatile Organics & 68 & 58 & 10 & 79 & Unacceptable Recoveries \\
\hline Semivolatile Organics & 64 & 60 & 4 & 94 & Unacceptable Recoveries \\
\hline Pesticides & 26 & 26 & 0 & 100 & - \\
\hline PCBs & 14 & 0 & 14 & 0 & Unacceptable RPD \\
\hline Dioxins and Furans & 34 & 34 & 0 & 100 & - \\
\hline Physical Properties & 4 & 2 & 2 & 50 & Density-Poor Precision \\
\hline Total & 524 & 460 & 64 & 88 & - \\
\hline
\end{tabular}

\section{A.1 Non-RCRA Metals}

Recoveries have been given in Table 4.2 for the non-RCRA metals. Tungsten, tantalum, and palladium method detection limits (MDLs) were determined but had expired prior to the sample analyses. In addition, boron was determined in the digestion method blank at $134 \mu \mathrm{g} / \mathrm{L}$, and laboratory control samples were outside of EPA SW-846 specifications for palladium and tantalum. With these exceptions and the recoveries noted in Table 4.2, non-RCRA metal analyses met all QC specifications. Detection limits have been given in Table 4.3.

The percent completeness for the non-RCRA metal analyses was $88 \%$. All palladium results were rejected during data validation because one of the two laboratory control sample recoveries was zero percent. Although this was expected to have been caused by not adding the palladium spike, this could not be verified. All tantalum results, lithium results from the peroxide-fusion samples, and uranium results from the peroxide fusion samples were considered to be invalid because recoveries were outside of specifications as noted in Table 4.2.

\section{A.2 RCRA Metals}

Recoveries have been given in Table 4.4 for the RCRA metals. Both antimony interference check results (124\% and $127 \%)$ were above the acceptance range. In addition, the initial interference check for beryllium (122\%) was above the acceptance range, and the final interference check for thallium (121\%) was above the acceptance range. These failures indicated the presence of another element could have produced a slightly high bias in the reported concentrations for antimony, beryllium, and thallium. Method 6010B indicates that such interferences should be handled by adjusting the results appropriately. Because antimony, beryllium, and thallium were reported as "nondetects" and because the effect of these interferences would be a high bias, these results were accepted without correction as conservative 
results. With these exceptions and the recoveries noted in Table 4.4, RCRA metal analyses met all QC specifications. Detection limits have been given in Table 4.5.

Table 4.2. Recoveries and RPD Values for Non-RCRA Metals

\begin{tabular}{|c|c|c|c|c|c|c|}
\hline & \multicolumn{3}{|c|}{ Acid Dissolution (\%) } & \multicolumn{3}{|c|}{ Peroxide Fusion (\%) } \\
\hline & Matrix Spike & Matrix Spike Dup & ${ }^{\#} \mathrm{RPD}$ & Matrix Spike & Matrix Spike Dup & ${ }^{\#} \mathrm{RPD}$ \\
\hline Al & 116 & 124.5 & 1.2 & 109 & 108 & 0.4 \\
\hline $\mathbf{B}$ & - & - & - & - & - & - \\
\hline $\mathbf{B i}$ & 105 & 105 & 0.3 & 107.5 & 123 & 14 \\
\hline $\mathbf{C a}$ & 108 & 124 & 1.7 & 96 & 94 & 0.5 \\
\hline Co & 97.5 & 98 & 1.1 & 98 & 98 & 0.3 \\
\hline $\mathbf{C u}$ & 102 & 103 & 0.9 & 101 & 101 & 0.2 \\
\hline $\mathbf{F e}$ & 96 & 110 & 0.3 & 96 & 94.5 & 0.4 \\
\hline $\mathbf{K}$ & 98.5 & 97 & 1.6 & 98 & 98 & 0.2 \\
\hline $\mathbf{L i}$ & 109 & 110 & 0.4 & $* * 129$ & 122.5 & 1.9 \\
\hline Mg & 91 & 91.5 & 0.2 & 89 & 90 & 0.9 \\
\hline Mn & 94 & 95 & 1.0 & 95 & 95 & 0.1 \\
\hline Mo & 110 & 108 & 1.7 & 118 & 113.5 & 3.8 \\
\hline $\mathrm{Na}$ & 116 & 117 & 0.1 & - & - & 0.4 \\
\hline $\mathbf{P}$ & 109 & 107.5 & 1.4 & 108 & 106 & 1.6 \\
\hline Pd & 109 & $* * 127$ & 15 & 88 & 94 & 4.6 \\
\hline $\mathbf{P t}$ & 112 & 105 & 6.4 & 105 & 111 & 6.0 \\
\hline $\mathbf{R h}$ & 100 & 102 & 2.1 & 101 & 105 & 4.4 \\
\hline $\mathbf{S}$ & 100 & 103 & 2.0 & 104 & 98 & 4.3 \\
\hline $\mathbf{S i}$ & $* 88$ & - & - & - & - & - \\
\hline $\mathbf{S n}$ & 110 & 110.5 & 0.5 & 121 & 108 & 11 \\
\hline $\mathbf{T a}$ & $* * 63$ & 75 & 13.5 & 87 & $* * 62$ & 34 \\
\hline $\mathbf{U}$ & 110 & 111 & 1.1 & 115 & $* * 129$ & 12 \\
\hline $\mathbf{W}$ & 107 & 105 & 2.5 & 92 & 102 & 11 \\
\hline $\mathbf{Y}$ & 110 & 103 & 6.3 & 114 & 108 & 4.7 \\
\hline $\mathbf{Z r}$ & 109 & 119 & 2.5 & 120 & 113 & 1.4 \\
\hline I & - & - & - & 100 & 99 & 1.0 \\
\hline
\end{tabular}

*This was the laboratory control sample recovery. No matrix spike was available for silicon or boron.

**These values were outside of the specified control limits of 75 to $125 \%{ }^{1}$

${ }^{\#}$ Specification for the relative percent difference was less than $20 \%{ }^{1}$

-Matrix spike and matrix spike duplicates were not performed for these analytes. 
WSRC-TR-2001-00584, Revision 0

SRT-RPP-2001-00220, Revision 0

Table 4.3. Detection and Quantitation Limits for Non-RCRA Metals

\begin{tabular}{|c|c|c|c|c|c|c|}
\hline & \multicolumn{2}{|c|}{ Method Limits $(\mu \mathrm{g} / \mathrm{L})$} & \multicolumn{4}{|c|}{ Sample Limits $(\mu \mathrm{g} / \mathrm{kg})$} \\
\hline & \multirow[b]{2}{*}{ DL } & \multirow[b]{2}{*}{ EQL } & \multicolumn{2}{|c|}{ Acid } & \multicolumn{2}{|c|}{ Fusion } \\
\hline & & & DL & EQL & DL & EQL \\
\hline Al & 28 & $2.8 \times 10^{2}$ & $1.1 \times 10^{5}$ & $1.1 \times 10^{6}$ & $5.6 \times 10^{5}$ & $5.6 \times 10^{6}$ \\
\hline B & 2.6 & 26 & $1.0 \times 10^{4}$ & $1.0 \times 10^{5}$ & $1.0 \times 10^{4}$ & $1.0 \times 10^{5}$ \\
\hline $\mathbf{B i}$ & $1.5 \times 10^{2}$ & $1.5 \times 10^{3}$ & $6.0 \times 10^{5}$ & $6.0 \times 10^{6}$ & $3.0 \times 10^{6}$ & $3.0 \times 10^{7}$ \\
\hline $\mathbf{C a}$ & 53 & $5.3 \times 10^{2}$ & $2.1 \times 10^{5}$ & $2.1 \times 10^{6}$ & $1.1 \times 10^{6}$ & $1.1 \times 10^{7}$ \\
\hline Co & 3.0 & 30 & $1.2 \times 10^{4}$ & $1.2 \times 10^{5}$ & $6.0 \times 10^{4}$ & $6.0 \times 10^{5}$ \\
\hline $\mathbf{C u}$ & 4.0 & 40 & $1.6 \times 10^{4}$ & $1.6 \times 10^{5}$ & $8.0 \times 10^{4}$ & $8.0 \times 10^{5}$ \\
\hline $\mathbf{F e}$ & 14 & $1.4 \times 10^{2}$ & $5.6 \times 10^{4}$ & $5.6 \times 10^{5}$ & $2.8 \times 10^{5}$ & $2.8 \times 10^{6}$ \\
\hline Mg & 26 & $2.6 \times 10^{2}$ & $1.0 \times 10^{5}$ & $1.0 \times 10^{6}$ & $5.2 \times 10^{5}$ & $5.2 \times 10^{6}$ \\
\hline Mn & 1.0 & 10 & $4.0 \times 10^{3}$ & $4.0 \times 10^{4}$ & $2.0 \times 10^{4}$ & $2.0 \times 10^{5}$ \\
\hline $\mathbf{K}$ & $1.2 \times 10^{2}$ & $1.2 \times 10^{3}$ & $4.8 \times 10^{5}$ & $4.8 \times 10^{6}$ & $2.4 \times 10^{6}$ & $2.4 \times 10^{7}$ \\
\hline $\mathrm{Na}$ & 26 & $2.6 \times 10^{2}$ & $1.0 \times 10^{5}$ & $1.0 \times 10^{6}$ & $5.2 \times 10^{5}$ & $5.2 \times 10^{6}$ \\
\hline Mo & 10 & $1.0 \times 10^{2}$ & $4.0 \times 10^{4}$ & $4.0 \times 10^{5}$ & $4.0 \times 10^{4}$ & $4.0 \times 10^{5}$ \\
\hline $\mathbf{L i}$ & 9.1 & 91 & $3.6 \times 10^{4}$ & $3.6 \times 10^{5}$ & $3.6 \times 10^{4}$ & $3.6 \times 10^{5}$ \\
\hline $\mathbf{P}$ & 34 & $3.4 \times 10^{2}$ & $1.4 \times 10^{5}$ & $1.4 \times 10^{6}$ & $1.4 \times 10^{5}$ & $1.4 \times 10^{6}$ \\
\hline Pd & $3.4 \times 10^{2}$ & $3.4 \times 10^{3}$ & $1.4 \times 10^{6}$ & $1.4 \times 10^{7}$ & $6.8 \times 10^{6}$ & $6.8 \times 10^{7}$ \\
\hline $\mathbf{P t}$ & 28 & $2.8 \times 10^{2}$ & $1.1 \times 10^{5}$ & $1.1 \times 10^{6}$ & $5.6 \times 10^{5}$ & $5.6 \times 10^{6}$ \\
\hline $\mathbf{R h}$ & $4.0 \times 10^{2}$ & $4.0 \times 10^{3}$ & $1.6 \times 10^{6}$ & $1.6 \times 10^{7}$ & $8.0 \times 10^{6}$ & $8.0 \times 10^{7}$ \\
\hline $\mathbf{S}$ & 22 & $2.2 \times 10^{2}$ & $8.8 \times 10^{4}$ & $8.8 \times 10^{5}$ & $8.8 \times 10^{4}$ & $8.8 \times 10^{5}$ \\
\hline $\mathbf{S i}$ & 47 & $4.7 \times 10^{2}$ & $1.9 \times 10^{5}$ & $1.9 \times 10^{6}$ & $1.9 \times 10^{6}$ & $1.9 \times 10^{7}$ \\
\hline Sn & 36 & $3.6 \times 10^{2}$ & $1.4 \times 10^{5}$ & $1.4 \times 10^{6}$ & $1.4 \times 10^{5}$ & $1.4 \times 10^{6}$ \\
\hline Ta & $1.5 \times 10^{2}$ & $1.5 \times 10^{3}$ & $6.0 \times 10^{5}$ & $6.0 \times 10^{6}$ & $6.0 \times 10^{5}$ & $6.0 \times 10^{6}$ \\
\hline $\mathbf{U}$ & $3.9 \times 10^{3}$ & $3.9 \times 10^{4}$ & $1.6 \times 10^{7}$ & $1.6 \times 10^{8}$ & $7.8 \times 10^{7}$ & $7.8 \times 10^{8}$ \\
\hline W & 67 & $6.7 \times 10^{2}$ & $2.7 \times 10^{5}$ & $2.7 \times 10^{6}$ & $2.7 \times 10^{5}$ & $2.7 \times 10^{6}$ \\
\hline $\mathbf{Y}$ & 15 & $1.5 \times 10^{2}$ & $6.0 \times 10^{4}$ & $6.0 \times 10^{5}$ & $3.0 \times 10^{5}$ & $3.0 \times 10^{6}$ \\
\hline $\mathrm{Zr}$ & 76 & $7.6 \times 10^{2}$ & $3.0 \times 10^{5}$ & $3.0 \times 10^{6}$ & $3.0 \times 10^{5}$ & $3.0 \times 10^{6}$ \\
\hline I & $3.0 \times 10^{2}$ & $3.0 \times 10^{3}$ & $6.0 \times 10^{4}$ & $6.0 \times 10^{5}$ & $6.0 \times 10^{4}$ & $6.0 \times 10^{5}$ \\
\hline
\end{tabular}

The percent completeness for the RCRA metal analyses was $93 \%$. Antimony results from the acid-dissolved samples and selenium results from atomic absorption measurements of the peroxide-fusion samples were considered invalid because recoveries were outside of specifications as noted in Table 4.4. Because Method 6010B does not call for rejection of results when interferences are possible, and because uncorrected results would be biased high, potential interferences discussed in the previous paragraph did not cause results to be considered invalid. 
WSRC-TR-2001-00584, Revision 0

SRT-RPP-2001-00220, Revision 0

Table 4.4. Recoveries for RCRA Metals

\begin{tabular}{|c|c|c|c|c|c|c|c|}
\hline & \multicolumn{3}{|c|}{ Acid Dissolution (\%) } & \multicolumn{3}{c|}{ Peroxide Fusion (\%) } \\
\hline & Matrix & Matrix Duplicate & RPD & Matrix & Matrix Duplicate & RPD \\
\hline$* * *$ Sb & 98.3 & $* 65.8$ & $* * 40$ & 94.7 & 92.2 & 2.7 \\
\hline As(AA) & 101.1 & 104.7 & 3.4 & 103.3 & 105.3 & 1.9 \\
\hline As (ES) & 97.8 & 97.0 & 0.8 & 96.3 & 97.0 & 0.7 \\
\hline $\mathbf{B a}$ & 97.2 & 97.6 & 0.4 & 96.1 & 96.5 & 0.4 \\
\hline$* * * \mathbf{B e}$ & 97.5 & 98.3 & 0.8 & 99.0 & 98.7 & 0.3 \\
\hline $\mathbf{C d}$ & 96.3 & 97.3 & 1.0 & 97.6 & 97.6 & 0.1 \\
\hline $\mathbf{C r}$ & 92.7 & 93.9 & 1.1 & 93.5 & 94.2 & 0.7 \\
\hline $\mathbf{P b}$ & 98.5 & 99.1 & 0.6 & 94.3 & 93.9 & 0.3 \\
\hline $\mathbf{H g}$ & 93.4 & 103.4 & 10 & - & - & - \\
\hline $\mathbf{N i}$ & 100.4 & 101.4 & 0.9 & - & - & - \\
\hline Se(AA) & 90.7 & 92.2 & 1.5 & 74.1 & 77.5 & 4.4 \\
\hline $\mathbf{S e} \mathbf{( E S )}$ & 101.5 & 101.0 & 0.5 & 100.0 & 101.1 & 1.1 \\
\hline $\mathbf{A g}$ & 104.2 & 104.4 & 0.1 & 103.2 & 100.4 & 2.3 \\
\hline$* * * \mathbf{T l}$ & 108.4 & 107.1 & 1.2 & 111.7 & 113.1 & 1.2 \\
\hline $\mathbf{V}$ & 96.0 & 96.9 & 0.9 & 96.7 & 96.8 & - \\
\hline $\mathbf{Z n}$ & 97.1 & 102.0 & 0.7 & 99.3 & 99.6 & 0.1 \\
\hline
\end{tabular}

*This value was outside of the specified control limits of 75 to $125 \%$. ${ }^{1}$

**This value was above specification for the relative percent difference of less than $20 \%$. ${ }^{1}$

*** One of these interference checks did not meet USEPA SW-846 specifications (80 to 120\%).

\section{A.3 TCLP Analyses}

Recoveries have been given in Table 4.6 for the TCLP analyses. Barium was found in the extraction method blank at $1220 \mu \mathrm{g} / \mathrm{L}$. The low-level concentration standards for the atomic absorption methods were $31 \%$ for mercury, $70 \%$ for arsenic, and $66 \%$ for selenium. Although USEPA SW-846 does not have a requirement for these low-level standards, they have been noted here as being outside of the QAPjP specification for recoveries (75\% to $125 \%$ ). Except for these low-level concentration standard results and the arsenic recovery noted in Table 4.6, TCLP analyses met all QC specifications. Detection and quantitation limits have also been given in Table 4.6.

The percent completeness for the TCLP analyses was $93 \%$. The arsenic result from the atomic absorption measurement was considered invalid because the matrix spike was outside of specifications as noted in Table 4.6. Although the low-level concentration standards for some of the analytes were low, calibrations did meet USEPA SW-846 requirements and did not cause the results to be considered invalid. 
WSRC-TR-2001-00584, Revision 0

SRT-RPP-2001-00220, Revision 0

Table 4.5. Detection and Quantitation Limits for RCRA Metals

\begin{tabular}{|c|c|c|c|c|c|c|}
\hline & \multicolumn{2}{|c|}{ Method Limits $(\mu \mathrm{g} / \mathrm{L})$} & \multicolumn{3}{|c|}{ Sample Limits $(\mu \mathrm{g} / \mathrm{kg})$} \\
\hline & & & \multicolumn{2}{|c|}{ Acid } & \multicolumn{2}{c|}{ Fusion } \\
\hline & DL & EQL & DL & EQL & DL & EQL \\
\hline $\mathbf{A g}$ & 7 & 70 & $2.8 \times 10^{4}$ & $2.8 \times 10^{5}$ & $1.4 \times 10^{5}$ & $1.4 \times 10^{6}$ \\
\hline $\mathbf{A s}(\mathbf{A A})$ & 0.41 & 4.1 & $1.6 \times 10^{3}$ & $1.6 \times 10^{4}$ & $8.2 \times 10^{3}$ & $8.2 \times 10^{4}$ \\
\hline $\mathbf{A s} \mathbf{( E S})$ & 17 & $1.7 \times 10^{2}$ & $6.8 \times 10^{4}$ & $6.8 \times 10^{5}$ & $3.4 \times 10^{5}$ & $3.4 \times 10^{6}$ \\
\hline $\mathbf{B a}$ & 2 & 20 & $8.0 \times 10^{3}$ & $8.0 \times 10^{4}$ & $4.0 \times 10^{4}$ & $4.0 \times 10^{5}$ \\
\hline $\mathbf{B e}$ & 0.4 & 4.0 & $1.6 \times 10^{3}$ & $1.6 \times 10^{4}$ & $8.0 \times 10^{3}$ & $8.0 \times 10^{4}$ \\
\hline $\mathbf{C d}$ & 2 & 20 & $8.0 \times 10^{3}$ & $8.0 \times 10^{4}$ & $4.0 \times 10^{4}$ & $4.0 \times 10^{5}$ \\
\hline $\mathbf{C r}$ & 8 & 80 & $3.2 \times 10^{4}$ & $3.2 \times 10^{5}$ & $1.6 \times 10^{5}$ & $1.6 \times 10^{6}$ \\
\hline $\mathbf{H g}$ & 0.1 & 1.0 & 20 & $2.0 \times 10^{2}$ & 20 & $2.0 \times 10^{2}$ \\
\hline $\mathbf{N i}$ & 4 & 40 & $1.6 \times 10^{4}$ & $1.6 \times 10^{5}$ & $8.0 \times 10^{4}$ & $8.0 \times 10^{5}$ \\
\hline $\mathbf{P b}$ & 38 & $3.8 \times 10^{2}$ & $1.5 \times 10^{5}$ & $1.5 \times 10^{6}$ & $7.6 \times 10^{5}$ & $7.6 \times 10^{6}$ \\
\hline $\mathbf{S b}$ & 35 & $3.5 \times 10^{2}$ & $1.4 \times 10^{5}$ & $1.4 \times 10^{6}$ & $7.0 \times 10^{5}$ & $7.0 \times 10^{6}$ \\
\hline $\mathbf{S e}(\mathbf{A A})$ & 0.74 & 7.4 & $2.9 \times 10^{3}$ & $2.9 \times 10^{4}$ & $1.5 \times 10^{4}$ & $1.5 \times 10^{5}$ \\
\hline $\mathbf{S e} \mathbf{( E S )}$ & 22 & $2.2 \times 10^{2}$ & $8.8 \times 10^{4}$ & $8.8 \times 10^{5}$ & $4.4 \times 10^{5}$ & $4.4 \times 10^{6}$ \\
\hline $\mathbf{T l}$ & 73 & $7.3 \times 10^{2}$ & $2.9 \times 10^{5}$ & $2.9 \times 10^{6}$ & $1.5 \times 10^{6}$ & $1.5 \times 10^{7}$ \\
\hline $\mathbf{V}$ & 2 & 20 & $8.0 \times 10^{3}$ & $8.0 \times 10^{4}$ & $4.0 \times 10^{4}$ & $4.0 \times 10^{5}$ \\
\hline $\mathbf{Z n}$ & 5 & 50 & $2.0 \times 10^{4}$ & $2.0 \times 10^{5}$ & $1.0 \times 10^{5}$ & $1.0 \times 10^{6}$ \\
\hline
\end{tabular}

Table 4.6 Recoveries, and Detection and Quantitation Limits for the TCLP Extract Analyses

\begin{tabular}{|c|c|c|c|c|c|c|c|}
\hline & \multicolumn{3}{|c|}{ Recoveries (\%) } & \multicolumn{2}{c|}{ Method Limits $(\mu \mathbf{g} / \mathbf{L})$} & \multicolumn{2}{c|}{ Sample Limits( $\mu \mathbf{g} / \mathbf{L})$} \\
\hline & Matrix & Matrix Dup & RPD & DL & EQL & DL & EQL \\
\hline Ag & 80.6 & 85.8 & 6.3 & 7.0 & 70 & 7.8 & 78 \\
\hline As(ES) & 97.6 & 100.8 & 3.3 & 17 & $1.7 \times 10^{2}$ & 19 & $1.9 \times 10^{2}$ \\
\hline As(AA) & $* 69.6$ & 71.1 & 2.1 & 0.4 & 4 & 0.46 & 4.6 \\
\hline Ba & 84.4 & 88.1 & 2.3 & 2.0 & 20 & 2.2 & 22 \\
\hline Be & 90.4 & 91.8 & 1.5 & 0.4 & 4 & 0.44 & 4.4 \\
\hline $\mathbf{C d}$ & 87.0 & 88.4 & 1.5 & 2.0 & 20 & 2.2 & 22 \\
\hline $\mathbf{C r}$ & 94.2 & 97.0 & 3.0 & 8.0 & 80 & 8.9 & 89 \\
\hline $\mathbf{H g}$ & 86.6 & 85.4 & 1.4 & 0.10 & 1.0 & 0.10 & 1.0 \\
\hline $\mathbf{N i}$ & 89.6 & 91.1 & 1.6 & 4.0 & 40 & 4.4 & 44 \\
\hline $\mathbf{P b}$ & 87.3 & 93.5 & 6.9 & 38 & $3.8 \times 10^{2}$ & 42 & $4.2 \times 10^{2}$ \\
\hline Sb & 93.1 & 96.6 & 3.7 & 35 & $3.5 \times 10^{2}$ & 39 & $3.9 \times 10^{2}$ \\
\hline Se(ES) & 100.3 & 103.0 & 2.7 & 22 & $2.2 \times 10^{2}$ & 24 & $2.4 \times 10^{2}$ \\
\hline Se(AA) & 77.3 & 91.7 & 15.9 & 0.73 & 7.3 & 0.81 & 8.1 \\
\hline Tl & 94.0 & 94.3 & 0.3 & 73 & $7.3 \times 10^{2}$ & 81 & $8.1 \times 10^{2}$ \\
\hline
\end{tabular}

* This value was outside of the specified control limits of 75 to $125 \%{ }^{1}$ 


\section{B Inorganic Analytes Detected as Ionic Species}

Recoveries, reproducibility, and detection and quantitation limits have been given in Table 4.7 for species measured as anions in the peroxide fusion dissolved samples. Because of difficulties in implementing the sodium removal, samples were analyzed 102 days after sample collection. With these exceptions and the recoveries noted in Table 4.7, ionic species analyses met all QC specifications. Blank and standard results have been presented in Table 4.8 along with results from the standard.

Table 4.7. Method 9056 Recovery, Reproducibility, and Detection and Quantitation Limits

\begin{tabular}{|c||c|c||c||c|c||c|c|}
\hline \multicolumn{1}{|c||}{} & \multicolumn{2}{c|}{ Recoveries (\%) } & *Duplicate (\%) & \multicolumn{2}{c||}{ Method Limit (mg/L) } & \multicolumn{2}{c|}{ Sample Limit (mg/kg) } \\
\hline & MS & LCS & & DL & EQL & DL & EQL \\
\hline $\mathbf{B r}$ & $* * 147$ & 81 & - & 7.4 & 25 & $1.5 \times 10^{3}$ & $5.0 \times 10^{3}$ \\
\hline $\mathbf{F}$ & 93 & 77 & 2.1 & 2.1 & 25 & $4.2 \times 10^{2}$ & $5.0 \times 10^{3}$ \\
\hline $\mathbf{C l}$ & $* * 133$ & 80 & - & 2.2 & 25 & $4.4 \times 10^{2}$ & $5.0 \times 10^{3}$ \\
\hline $\mathbf{N O}_{\mathbf{3}}$ & 125 & 80 & - & 5.5 & 25 & $1.1 \times 10^{3}$ & $5.0 \times 10^{3}$ \\
\hline $\mathbf{N O}_{\mathbf{2}}$ & 108 & 78 & - & 4.9 & 25 & $9.8 \times 10^{2}$ & $5.0 \times 10^{3}$ \\
\hline $\mathbf{P O}_{4}$ & $* * 70$ & 84 & 14 & 4.6 & 25 & $9.2 \times 10^{2}$ & $5.0 \times 10^{3}$ \\
\hline $\mathbf{S O}_{4}$ & 76 & 84 & 0.9 & 4.0 & 25 & $8.0 \times 10^{2}$ & $5.0 \times 10^{3}$ \\
\hline
\end{tabular}

*These values were determined by performing duplicate analyses of the low-activity test reference material "field blank".

**These values were outside of the specified control limits of 75 to $125 \%{ }^{1}$

The percent completeness for the inorganic anion analyses was $70 \%$. Bromide, chloride, and phosphate results were considered invalid because the matrix spike recoveries were outside of specifications as noted in Table 4.7. Although analysis was 102 days after sample collection, this did not violate any specifications. In addition, these analytes are expected to have been stable in these samples, and samples gave no indication of degradation.

\section{C Radionuclide Analyses}

Minimum detectable activities (MDAs) have been given in Table 4.9 for the radionuclide analyses. Neptunium-237 results were initially rejected during data validation because an erroneous region of interest was used in the energy spectra. The spectra were re-evaluated by BWXT Services, and results from this evaluation have been given in this report. Carbon-14 results were rejected because of interference from other beta-emitting radionuclides. In addition, some niobium-94, europium-152, cesium-137, cobalt-60, and radium-226 results were rejected because one of the key energy peaks was not present. With these exceptions radionuclide analyses met all QC specifications. 
WSRC-TR-2001-00584, Revision 0

SRT-RPP-2001-00220, Revision 0

Table 4.8. Results from Method 9056 Analyses of Blanks and Standard

\begin{tabular}{|c||c|c|c|c|c|c|}
\hline & \multicolumn{3}{c|}{ Blanks $(\mathrm{mg} / \mathrm{kg})$} & \multicolumn{2}{c|}{ Standard (mg/kg) } \\
\hline & Method & \multicolumn{2}{|c|}{ Process } & ${ }^{* * * \text { Field }}$ & Measured & *Actual \\
\hline & & Measured & $* *$ Target & & & \\
\hline $\mathbf{B r}$ & $<5 \times 10^{3}$ & $<5 \times 10^{3}$ & - & $<5 \times 10^{3}$ & $<5 \times 10^{3}$ & - \\
\hline $\mathbf{C l}$ & $<5 \times 10^{3}$ & $<5 \times 10^{3}$ & $2.1 \times 10^{3}$ & $<5 \times 10^{3}$ & $<5 \times 10^{3}$ & - \\
\hline $\mathbf{F}$ & $<5 \times 10^{3}$ & $<5 \times 10^{3}$ & $6.4 \times 10^{2}$ & ${ }^{\mathrm{B}} 8.5 \times 10^{3}$ & ${ }^{\mathrm{B}} 8.5 \pm 0.56 \times 10^{3}$ & $8.6 \pm 0.35 \times 10^{3}$ \\
\hline $\mathbf{N O}_{3}$ & $<5 \times 10^{3}$ & $<5 \times 10^{3}$ & - & $<5 \times 10^{3}$ & $<5 \times 10^{3}$ & - \\
\hline $\mathbf{N O}_{2}$ & $<5 \times 10^{3}$ & $<5 \times 10^{3}$ & - & $<5 \times 10^{3}$ & $<5 \times 10^{3}$ & - \\
\hline $\mathbf{P O}_{4}$ & $<5 \times 10^{3}$ & $<5 \times 10^{3}$ & $9.6 \times 10^{2}$ & ${ }^{\mathrm{B}} 6.6 \times 10^{3}$ & ${ }^{\mathrm{B}} 6.3 \pm 0.43 \times 10^{3}$ & - \\
\hline $\mathbf{S O}_{4}$ & $<5 \times 10^{3}$ & ${ }^{\mathrm{J}} 4.3 \pm 0.31 \times 10^{3}$ & $3.5 \times 10^{3}$ & ${ }^{\mathrm{J}} 4.4 \times 10^{3}$ & ${ }^{\mathrm{J}} 4.1 \pm 0.19 \times 10^{3}$ & $3.6 \pm 0.59 \times 10^{3}$ \\
\hline
\end{tabular}

"<"indicates a result that was less than the EQL. This is usually indicated by a "U" qualifier.

${ }^{\mathrm{B}}$ Concentration was greater than the EQL but less than ten times EQL.

${ }^{\mathrm{J}}$ Analyte concentration was estimated because a peak was apparent but was below the EQL.

*These results were from an interlaboratory study of the low-activity test reference material glass standard. ${ }^{5}$

** These results were determined oxide composition specified by the Vitreous State Laboratory. ****The actual composition of the field "blank" is the same as the composition of the standard.

The percent completeness for the radionuclide analyses was $91 \%$. For the acid-dissolved samples, one of the uranium-236 results, one of the niobium-94 results, and one of the europium255 results were rejected. For the peroxide-fusion samples, one of the selenium-79 results, one of the uranium-235 results, and both of the uranium-236 results were rejected. All carbon-14 results were rejected. Reasons for rejecting these results have been given in the previous paragraph.

\section{D Organic Analyses}

Recoveries and quantitation limits have been given in Table 4.9 and 4.10 for the organic analyses. Triethylamine (TEA) recovery in the laboratory control sample (16\%) was below the acceptance limits. In addition, the instrument did not pass tuning requirements. Therefore, TEA results were rejected during data validation. Initial methanol and 2-butoxyethanol analyses did not contain surrogate standards. Analyses were repeated, but the samples had exceeded their hold times. With these exceptions and the recoveries noted in Table 4.9, organic analyses met all QC specifications.

The percent completeness values for the pesticide, and dioxin and furan analyses were $100 \%$. Because of low 1,4-dichlorobenzene and low triethylamine recoveries, the percent completeness for the semivolatile organic compound analyses was $94 \%$. Percent completeness for the PCBs was $0 \%$. Although PCB analyses met all other QA and QC requirements, the RPD values for the matrix spike and matrix duplicates did not meet specifications as shown in Table 4.10. 
WSRC-TR-2001-00584, Revision 0

SRT-RPP-2001-00220, Revision 0

Table 4.8. Minimum Detectable Activities

\begin{tabular}{|c|c|c|c|c|c|c|c|c|}
\hline & \multicolumn{4}{|c|}{ Method Limits (pCi/L) } & \multicolumn{4}{|c|}{ Sample Limits (pCi/kg) } \\
\hline & \multicolumn{2}{|c|}{ Acid Dissolution } & \multicolumn{2}{|c|}{ Peroxide Fusion } & \multicolumn{2}{|c|}{ Acid Dissolution } & \multicolumn{2}{|c|}{ Peroxide Fusion } \\
\hline Sample \# & AA1 & AA2 & FA1 & FA2 & AA1 & AA2 & FA1 & FA2 \\
\hline Alpha & $4.0 \times 10^{2}$ & $4.5 \times 10^{2}$ & $1.2 \times 10^{3}$ & $1.3 \times 10^{3}$ & $7.9 \times 10^{4}$ & $8.7 \times 10^{4}$ & $2.3 \times 10^{5}$ & $2.5 \times 10^{5}$ \\
\hline Beta & $1.9 \times 10^{2}$ & $1.9 \times 10^{2}$ & $1.2 \times 10^{3}$ & $1.1 \times 10^{3}$ & $3.8 \times 10^{4}$ & $3.7 \times 10^{4}$ & $2.2 \times 10^{5}$ & $2.2 \times 10^{5}$ \\
\hline Am-241 & 58 & 42 & 22 & 54 & $1.2 \times 10^{4}$ & $8.1 \times 10^{3}$ & $4.1 \times 10^{3}$ & $1.1 \times 10^{4}$ \\
\hline$* A m-241$ & $3.3 \times 10^{3}$ & $3.8 \times 10^{3}$ & $5.6 \times 10^{3}$ & $3.6 \times 10^{3}$ & $6.6 \times 10^{5}$ & $7.5 \times 10^{5}$ & $1.1 \times 10^{6}$ & $7.1 \times 10^{5}$ \\
\hline C-14 & $6.6 \times 10^{3}$ & $6.1 \times 10^{3}$ & $7.4 \times 10^{3}$ & $5.3 \times 10^{3}$ & $1.3 \times 10^{6}$ & $1.2 \times 10^{6}$ & $1.4 \times 10^{6}$ & $1.1 \times 10^{6}$ \\
\hline Cm-242 & 57 & 25 & 24 & 25 & $1.1 \times 10^{4}$ & $5.0 \times 10^{3}$ & $4.6 \times 10^{3}$ & $4.9 \times 10^{3}$ \\
\hline Cm-244 & 25 & 28 & 22 & 47 & $5.1 \times 10^{3}$ & $5.5 \times 10^{3}$ & $4.1 \times 10^{3}$ & $9.5 \times 10^{3}$ \\
\hline Co-60 & $5.5 \times 10^{2}$ & $6.5 \times 10^{2}$ & $7.4 \times 10^{2}$ & $3.7 \times 10^{2}$ & $1.1 \times 10^{5}$ & $1.3 \times 10^{5}$ & $1.4 \times 10^{5}$ & $7.4 \times 10^{4}$ \\
\hline Cs-134 & $6.9 \times 10^{2}$ & $5.1 \times 10^{2}$ & $8.5 \times 10^{2}$ & $5.2 \times 10^{2}$ & $1.4 \times 10^{5}$ & $1.0 \times 10^{5}$ & $1.6 \times 10^{5}$ & $1.0 \times 10^{5}$ \\
\hline Cs-137 & $1.2 \times 10^{3}$ & $4.8 \times 10^{2}$ & $8.4 \times 10^{2}$ & $4.1 \times 10^{2}$ & $2.5 \times 10^{5}$ & $9.3 \times 10^{4}$ & $1.6 \times 10^{5}$ & $8.1 \times 10^{4}$ \\
\hline Eu-152 & $2.1 \times 10^{3}$ & $1.6 \times 10^{3}$ & $2.5 \times 10^{3}$ & $2.2 \times 10^{3}$ & $4.2 \times 10^{5}$ & $3.2 \times 10^{5}$ & $4.7 \times 10^{5}$ & $4.4 \times 10^{5}$ \\
\hline Eu-154 & $1.8 \times 10^{3}$ & $1.8 \times 10^{3}$ & $2.8 \times 10^{3}$ & $1.2 \times 10^{3}$ & $3.5 \times 10^{5}$ & $3.4 \times 10^{5}$ & $5.3 \times 10^{5}$ & $2.4 \times 10^{5}$ \\
\hline Eu-155 & $3.3 \times 10^{3}$ & $2.7 \times 10^{3}$ & $3.3 \times 10^{3}$ & $2.6 \times 10^{3}$ & $6.7 \times 10^{5}$ & $5.2 \times 10^{5}$ & $6.1 \times 10^{5}$ & $5.1 \times 10^{5}$ \\
\hline H-3 & $1.1 \times 10^{4}$ & $1.1 \times 10^{4}$ & $1.1 \times 10^{4}$ & $1.1 \times 10^{4}$ & $2.2 \times 10^{6}$ & $2.2 \times 10^{6}$ & $2.1 \times 10^{6}$ & $2.2 \times 10^{6}$ \\
\hline I-129 & $1.9 \times 10^{3}$ & $9.8 \times 10^{2}$ & $1.8 \times 10^{3}$ & $4.0 \times 10^{2}$ & $3.8 \times 10^{5}$ & $1.9 \times 10^{5}$ & $3.4 \times 10^{5}$ & $7.9 \times 10^{4}$ \\
\hline $\mathrm{Nb}-94$ & $1.0 \times 10^{3}$ & $9.5 \times 10^{2}$ & $1.5 \times 10^{3}$ & $8.6 \times 10^{2}$ & $2.1 \times 10^{5}$ & $1.8 \times 10^{5}$ & $2.8 \times 10^{5}$ & $1.7 \times 10^{5}$ \\
\hline Np-237 & 22 & 18 & 21 & 20 & $4.3 \times 10^{3}$ & $3.4 \times 10^{3}$ & $3.9 \times 10^{3}$ & $4.0 \times 10^{3}$ \\
\hline Pu-238 & 22 & 53 & 21 & 51 & $4.3 \times 10^{3}$ & $1.0 \times 10^{4}$ & $3.9 \times 10^{3}$ & $1.0 \times 10^{4}$ \\
\hline Pu239/240 & 22 & 24 & 21 & 20 & $4.3 \times 10^{3}$ & $4.8 \times 10^{3}$ & $3.9 \times 10^{3}$ & $4.0 \times 10^{3}$ \\
\hline Pu-241 & $5.8 \times 10^{3}$ & $5.9 \times 10^{3}$ & $5.6 \times 10^{3}$ & $6.0 \times 10^{3}$ & $1.2 \times 10^{6}$ & $1.1 \times 10^{6}$ & $1.1 \times 10^{6}$ & $1.2 \times 10^{6}$ \\
\hline Ra-226 & $1.6 \times 10^{3}$ & $1.1 \times 10^{3}$ & $1.6 \times 10^{3}$ & $1.2 \times 10^{3}$ & $3.3 \times 10^{5}$ & $2.1 \times 10^{5}$ & $3.1 \times 10^{5}$ & $2.4 \times 10^{5}$ \\
\hline Ru-103 & $8.8 \times 10^{2}$ & $6.0 \times 10^{2}$ & $1.0 \times 10^{3}$ & $6.8 \times 10^{2}$ & $1.8 \times 10^{5}$ & $1.2 \times 10^{5}$ & $1.9 \times 10^{5}$ & $1.4 \times 10^{5}$ \\
\hline Ru-106 & $7.0 \times 10^{3}$ & $5.4 \times 10^{3}$ & $9.1 \times 10^{3}$ & $5.3 \times 10^{3}$ & $1.4 \times 10^{6}$ & $1.0 \times 10^{6}$ & $1.7 \times 10^{6}$ & $1.1 \times 10^{6}$ \\
\hline Se-79 & $3.5 \times 10^{3}$ & $3.0 \times 10^{4}$ & $3.0 \times 10^{4}$ & $8.9 \times 10^{4}$ & $7.0 \times 10^{5}$ & $5.9 \times 10^{6}$ & $5.5 \times 10^{6}$ & $1.8 \times 10^{7}$ \\
\hline Sr-90 & $4.9 \times 10^{2}$ & $3.9 \times 10^{2}$ & $4.8 \times 10^{2}$ & $5.7 \times 10^{2}$ & $9.7 \times 10^{4}$ & $7.7 \times 10^{4}$ & $9.0 \times 10^{4}$ & $1.1 \times 10^{5}$ \\
\hline Tc-99 & $4.6 \times 10^{2}$ & $4.4 \times 10^{2}$ & $4.2 \times 10^{2}$ & $4.0 \times 10^{2}$ & $9.2 \times 10^{4}$ & $8.5 \times 10^{4}$ & $7.9 \times 10^{4}$ & $8.0 \times 10^{4}$ \\
\hline U-234 & 5.5 & 8.8 & 4.3 & 5.5 & $1.1 \times 10^{3}$ & $1.7 \times 10^{3}$ & $8.0 \times 10^{2}$ & $1.1 \times 10^{3}$ \\
\hline U-235 & 5.5 & 6.0 & 4.3 & 5.5 & $1.1 \times 10^{3}$ & $1.2 \times 10^{3}$ & $8.0 \times 10^{2}$ & $1.1 \times 10^{3}$ \\
\hline U-236 & 5.5 & 4.6 & 4.8 & 5.5 & $1.1 \times 10^{3}$ & $8.9 \times 10^{2}$ & $8.9 \times 10^{2}$ & $1.1 \times 10^{3}$ \\
\hline U-238 & 5.5 & 5.1 & 4.3 & 5.5 & $1.1 \times 10^{3}$ & $9.9 \times 10^{2}$ & $8.0 \times 10^{2}$ & $1.1 \times 10^{3}$ \\
\hline
\end{tabular}

\section{E Physical Properties}

Results from the ignitability tests (USEPA SW-846, Method 1010) meet all QA and QC requirements. Density results have not been included in this report. Results from the duplicate analyses varied greatly. Evaluation of the data package showed use of relatively small samples caused a large discrepancy in the sample masses. Because sample was limited a small quantity of the sample was available for these measurements. The discrepancy appears to have been due to 
errors associated with measuring this sample mass as the difference between the container mass after the sample transferred and the empty container mass.

Table 4.9. Recoveries and Quantitation Limits for Volatile Organic Analyses

\begin{tabular}{|c|c|c|c||c|}
\hline \multicolumn{1}{|c|}{ Analyte } & \multicolumn{3}{|c||}{ Recoveries (\%) } & EQL $(\mu \mathrm{g} / \mathbf{k g})$ \\
\hline Acetone & Matrix & Duplicate & RPD & \\
\hline 2-Butanol & 116 & - & - & 10 \\
\hline 2-Butanone & - & - & - & 100 \\
\hline 2-Butenal & $* 131$ & - & - & 10 \\
\hline Chloromethane & 106 & - & - & 10 \\
\hline 1,2-Dichloroethane & 68 & - & - & 10 \\
\hline Ethylene Oxide & 107 & - & - & 10 \\
\hline Isopropanol & 85 & - & - & 10 \\
\hline Methylene Chloride & 90 & - & - & 100 \\
\hline 2-Methyl-2-Propanol & 118 & - & - & 10 \\
\hline Tetrahydrofuran & 96 & - & - & 100 \\
\hline Vinyl Chloride & 102 & - & - & 10 \\
\hline 2-Butoxyethanol & 66 & - & - & 10 \\
\hline Methanol & 72 & 92 & 24 & See Results Chapter \\
\hline
\end{tabular}

*This value was outside of the specified control limits of 50 to $130 \%{ }^{1}$

\subsection{Results}

Results from the analyses have been presented in this chapter. Results have been given for all "target" analytes (analytes specified in the QAPjP for this task) ${ }^{1}$ and for other analytes detected during these analyses. For target analytes that were not detected, MDLs have been present with a "<" designation. If a result was found to be a "nondetect" during data validation, it has been presented with a "U" data qualifier. Similarly, results between the MDL and EQL have been given a "B" data qualifier (MDL and EQL values were determined according to USEPA SW-846 protocol and have been given in the previous chapter). If the result was considered as an estimate for another reason, the result has been given a "J" data qualifier. If the matrix spike or matrix spike duplicate recoveries, or the relative percent difference between these values fell outside of the specified limits, the result has been given an "N" data qualifier. ${ }^{1}$ 
WSRC-TR-2001-00584, Revision 0

SRT-RPP-2001-00220, Revision 0

Table 4.10. Recoveries and Quantitation Limits for Miscellaneous Organic Analyses

\begin{tabular}{|c|c|c|c|c|c|}
\hline \multirow[t]{2}{*}{ Analysis } & \multirow[t]{2}{*}{ Analyte } & \multicolumn{3}{|c|}{ Recoveries (\%) } & \multirow[t]{2}{*}{ Quantitation $(\mu \mathrm{g} / \mathrm{kg})$} \\
\hline & & Matrix & Duplicate & RPD & \\
\hline \multirow{5}{*}{ Semivolatile } & Phenol & 79 & 65 & 19 & \multirow{5}{*}{$\begin{array}{l}\text { See Results Chapter } \\
\text { for Target } \\
\text { Semivolatile Organic } \\
\text { Compound EQLs. }\end{array}$} \\
\hline & 1,4-Dichlorobenzene & 78 & 58 & $* * 29$ & \\
\hline & 2,4-Dinitrotoluene & 94 & 82 & 14 & \\
\hline & Pentachlorophenol & 88 & 82 & 7 & \\
\hline & 1,2,4-Trichlorobenzene & 84 & 67 & 22 & \\
\hline \multirow{6}{*}{ Pesticides } & Aldrin & 72 & 84 & 15 & \multirow{6}{*}{$\begin{array}{l}\text { See Results Chapter } \\
\text { for Pesticide EQLs. }\end{array}$} \\
\hline & Gamma-BHC (Lindane) & 81 & 100 & 21 & \\
\hline & 4,4'-DDT & 77 & 95 & 21 & \\
\hline & Dieldrin & 82 & 98 & 18 & \\
\hline & Endrin & 108 & $* 131$ & 19 & \\
\hline & Heptachlor & 81 & 94 & 15 & \\
\hline \multirow{2}{*}{ PCBs } & Aroclor-1016 & 115 & 88 & $* * 27$ & \multirow{2}{*}{$\begin{array}{l}\text { See Results Chapter } \\
\text { for PCB EQLs.) }\end{array}$} \\
\hline & Aroclor-1260 & 118 & 92 & $* * 25$ & \\
\hline \multirow{17}{*}{$\begin{array}{l}\text { Dioxins } \\
\text { And } \\
\text { Furans }\end{array}$} & 2378-TCDD & 72 & 64 & 12 & 0.2 \\
\hline & 12378-PeCDD & 97 & 87 & 10 & 0.2 \\
\hline & 123478-HxCDD & 106 & 92 & 14 & 0.3 \\
\hline & 123678-HxCDD & 110 & 102 & 7.5 & 0.3 \\
\hline & 123789-HxCDD & 110 & 117 & 6.2 & 0.3 \\
\hline & 1234678-HpCDD & 92 & 83 & 9.8 & 0.4 \\
\hline & OCDD & 96 & 90 & 5.7 & 0.6 \\
\hline & 2378-TCDF & 101 & 92 & 9.2 & 0.3 \\
\hline & 12378-PeCDF & 111 & 98 & 12 & 0.2 \\
\hline & 23478-PeCDF & 122 & 108 & 12 & 0.2 \\
\hline & 123478-HxCDF & 101 & 92 & 9.8 & 0.2 \\
\hline & 123678-HxCDF & 102 & 93 & 9.2 & 0.2 \\
\hline & 234678-HxCDF & 88 & 82 & 6.8 & 0.2 \\
\hline & 123789-HxCDF & 88 & 94 & 7.5 & 0.2 \\
\hline & 1234678-HpCDF & 92 & 84 & 8.2 & 0.2 \\
\hline & 1234789-HpCDF & 100 & 94 & 6.4 & 0.3 \\
\hline & OCDF & 101 & 96 & 5.5 & 0.5 \\
\hline
\end{tabular}

*This value was outside of the specified control limits of 50 to $130 \%$. ${ }^{1}$

**This value was above specification for the relative percent deviation of less than $25 \%{ }^{1}$ 


\section{A Metals}

Results given in this section are the concentrations of the metal species in the glass waste form. These results have been separated into non-RCRA metal results and RCRA metal results. Also presented in this section are comparisons of these USPA SW-846 results for the AN-102 melter glass to results determined at SRTC from similar samples and to crucible-scale vitrification tests performed on the AN-102 material. This section also includes results from TCLP tests on the melter-scale AN-102 glass waste form.

\section{A.1 Measured Composition}

Results from the ICP-ES analyses (Method 6010B), and the AA analyses (Method 7060A for arsenic, Method 7470A for mercury, and Method 7740 for selenium) are presented in this section. In addition, the average for each of the analytes has been given. To be conservative, for analytes that were detected in one sample but not in the other, the detected value was given as the average concentration.

\section{A.1.a. Non-RCRA Metals}

Concentrations have been given in Table 5.1 for the non-RCRA metals. These values showed

-For all analytes except tantalum, results from at least one dissolution method met all QC requirements.

-Average acid and average fusion results were within $20 \%$ for primary analytes except lithium.

-Conversion of these results to percent on an oxide basis showed $100 \%$ of the waste form mass was accounted for by the analytes present at concentrations above the EQL.

Results in Table 5.1 indicate that for analytes detected in both sets of samples, results were similar (within $20 \%$ ) for all primary analytes (those present at more than $10^{7} \mu \mathrm{g} / \mathrm{kg}$ ) except lithium. Except phosphorous and palladium, all results above the detection limits were higher for the peroxide fusion samples than the acid samples. This may have been caused by small errors in measuring sample mass, volume transfers, or dilutions.

Matrix spike recovery was high for lithium (129\%). Although the matrix spike duplicate recovery was within the specified range, it too was high $(122.5 \%)$. High lithium recoveries in the fusion samples appear to have been the cause for the discrepancy between the lithium results for the acid-dissolved samples and the fusion samples. In addition, some detection limits were high. Potassium limits can be lowered by using USEPA SW-846 Method 7610 instead of Method 6010B. The other detection limits may be lowered if the samples can be analyzed without the sample digestion (Method 3015).

In addition, some detection limits were high. Potassium limits can be lowered by using USEPA SW-846 Method 7610 instead of Method 6010B. The other detection limits may be lowered if the samples can be analyzed without the sample digestion (Method 3015). 
WSRC-TR-2001-00584, Revision 0

SRT-RPP-2001-00220, Revision 0

Table 5.1. Concentrations of Target Non-RCRA Metal Analytes Measured by ICP-ES and AA

\begin{tabular}{|c|c|c|c|c|c|c|}
\hline & \multicolumn{3}{|c|}{ Acid Dissolution $(\mu \mathrm{g} / \mathrm{kg})$} & \multicolumn{3}{|c|}{ Peroxide Fusion $(\mu \mathrm{g} / \mathrm{kg})$} \\
\hline & AA1 & AA2 & Average & FA1 & FA2 & Average \\
\hline $\mathrm{Al}$ & $2.4 \times 10^{7}$ & $2.8 \times 10^{7}$ & $2.6 \pm 0.30 \times 10^{7}$ & $2.95 \times 10^{7}$ & $2.87 \times 10^{7}$ & $2.91 \pm 0.051 \times 10^{7}$ \\
\hline $\mathrm{Ca}$ & $3.3 \times 10^{7}$ & $4.0 \times 10^{7}$ & $3.7 \pm 0.47 \times 10^{7}$ & $4.6 \times 10^{7}$ & $4.3 \times 10^{7}$ & $4.4 \pm 0.11 \times 10^{7}$ \\
\hline $\mathrm{Co}$ & $<1 \times 10^{4}$ & $<1 \times 10^{4}$ & $<1 \times 10^{4}$ & ${ }^{\mathrm{B}} 2.8 \times 10^{5}$ & ${ }^{\mathrm{B}} 1.3 \times 10^{5}$ & $\mathrm{~B}_{2.0 \pm 1.0 \times 10^{5}}$ \\
\hline $\mathrm{Cu}$ & $2.1 \times 10^{5}$ & $2.4 \times 10^{5}$ & $2.3 \pm 0.19 \times 10^{5}$ & $\mathrm{~B}_{3.8 \times 10^{5}}$ & ${ }^{\mathrm{B}} 2.9 \times 10^{5}$ & ${ }^{B} 3.3 \pm 0.69 \times 10^{5}$ \\
\hline $\mathrm{Fe}$ & $3.97 \times 10^{7}$ & $4.08 \times 10^{7}$ & $4.03 \pm 0.077 \times 10^{7}$ & $4.3 \times 10^{7}$ & $4.1 \times 10^{7}$ & $4.2 \pm 0.15 \times 10^{7}$ \\
\hline $\mathrm{Mg}$ & $7.7 \times 10^{6}$ & $8.0 \times 10^{6}$ & $7.8 \pm 0.20 \times 10^{6}$ & $8.6 \times 10^{6}$ & $8.2 \times 10^{6}$ & $8.4 \pm 0.28 \times 10^{6}$ \\
\hline $\mathrm{Mn}$ & $2.08 \times 10^{5}$ & $2.14 \times 10^{5}$ & $2.11 \pm 0.044 \times 10^{5}$ & $1.8 \times 10^{6}$ & $9.9 \times 10^{5}$ & $1.4 \pm 0.54 \times 10^{6}$ \\
\hline $\mathrm{K}$ & ${ }^{\mathrm{B}} 1.24 \times 10^{6}$ & ${ }^{\mathrm{B}} 1.16 \times 10^{6}$ & ${ }^{\mathrm{B}} 1.20 \pm 0.059 \times 10^{6}$ & $*$ & $*$ & $*$ \\
\hline $\mathrm{Na}$ & $7.15 \times 10^{7}$ & $7.23 \times 10^{7}$ & $7.19 \pm 0.059 \times 10^{7}$ & $*$ & $*$ & $*$ \\
\hline Mo & ${ }^{\mathrm{B}} 4.5 \times 10^{4}$ & $<4 \times 10^{4}$ & ${ }^{B} 4.5 \times 10^{4}$ & $<4 \times 10^{4}$ & $<4 \times 10^{4}$ & $<4 \times 10^{4}$ \\
\hline $\mathrm{Li}$ & $1.27 \times 10^{7}$ & $1.32 \times 10^{7}$ & $1.30 \pm 0.034 \times 10^{7}$ & $\mathrm{~N}_{1.68 \times 10^{7}}$ & $\mathrm{~N}_{1.65 \times 10^{7}}$ & ${ }^{\mathrm{N}} 1.67 \pm 0.021 \times 10^{7}$ \\
\hline $\mathrm{P}$ & ${ }^{\mathrm{B}} 7.0 \times 10^{5}$ & ${ }^{\mathrm{B}} 6.4 \times 10^{5}$ & ${ }^{\mathrm{B}} 6.7 \pm 0.39 \times 10^{5}$ & ${ }^{\mathrm{B}} 6.2 \times 10^{5}$ & ${ }^{\mathrm{B}} 6.5 \times 10^{5}$ & ${ }^{\mathrm{B}} 6.3 \pm 0.20 \times 10^{5}$ \\
\hline $\mathrm{Zr}$ & $2.42 \times 10^{7}$ & $2.45 \times 10^{7}$ & $2.43 \pm 0.024 \times 10^{7}$ & $2.73 \times 10^{7}$ & $2.61 \times 10^{7}$ & $2.67 \pm 0.082 \times 10^{7}$ \\
\hline $\mathrm{S}$ & $1.38 \times 10^{6}$ & $1.33 \times 10^{6}$ & $1.36 \pm 0.037 \times 10^{6}$ & $1.6 \times 10^{6}$ & $1.4 \times 10^{6}$ & $1.5 \pm 0.14 \times 10^{6}$ \\
\hline $\mathrm{Sn}$ & $<1 \times 10^{5}$ & $<1 \times 10^{5}$ & $<1 \times 10^{5}$ & $<1 \times 10^{5}$ & $<1 \times 10^{5}$ & $<1 \times 10^{5}$ \\
\hline $\mathrm{Si}$ & $2.26 \times 10^{8}$ & $2.22 \times 10^{8}$ & $2.24 \pm 0.028 \times 10^{8}$ & $2.41 \times 10^{8}$ & $2.39 \times 10^{8}$ & $2.40 \pm 0.012 \times 10^{8}$ \\
\hline B & $*$ & $*$ & $*$ & $3.68 \times 10^{7}$ & $3.67 \times 10^{7}$ & $3.67 \pm 0.008 \times 10^{7}$ \\
\hline $\mathrm{W}$ & $<3 \times 10^{5}$ & $<3 \times 10^{5}$ & $<3 \times 10^{5}$ & $<3 \times 10^{5}$ & $<3 \times 10^{5}$ & $<3 \times 10^{5}$ \\
\hline $\mathrm{Ta}$ & ${ }^{\mathrm{BN}} 7.9 \times 10^{5}$ & ${ }^{\mathrm{BN}} 1.4 \times 10^{6}$ & ${ }^{\mathrm{BN}} 1.1 \pm 0.46 \times 10^{6}$ & $\mathrm{~N}_{<6 \times 10^{5}}$ & $\mathrm{~N}_{<6 \times 10^{5}}$ & $\mathrm{~N}_{<6 \times 10^{5}}$ \\
\hline $\mathrm{Bi}$ & $<6 \times 10^{5}$ & $<6 \times 10^{5}$ & $<6 \times 10^{5}$ & $<3 \times 10^{6}$ & $<3 \times 10^{6}$ & $<3 \times 10^{6}$ \\
\hline $\mathrm{Pt}$ & $<1 \times 10^{5}$ & $<1 \times 10^{5}$ & $<1 \times 10^{5}$ & $<5 \times 10^{5}$ & $<6 \times 10^{5}$ & $<5 \times 10^{5}$ \\
\hline $\mathrm{Y}$ & $<6 \times 10^{4}$ & $<6 \times 10^{4}$ & $<6 \times 10^{4}$ & $<3 \times 10^{5}$ & $<3 \times 10^{5}$ & $<3 \times 10^{5}$ \\
\hline $\mathrm{Pd}$ & $\mathrm{N}_{<1 \times 10^{6}}$ & ${ }^{\mathrm{BN}} 1.0 \times 10^{7}$ & ${ }^{\mathrm{BN}} 1.0 \times 10^{7}$ & ${ }^{\mathrm{B}} 1.0 \times 10^{7}$ & ${ }^{\mathrm{B}} 8.2 \times 10^{6}$ & ${ }^{\mathrm{B}} 9.2 \pm 1.4 \times 10^{6}$ \\
\hline $\mathrm{Rh}$ & $<2 \times 10^{6}$ & $<2 \times 10^{6}$ & $<2 \times 10^{6}$ & $<8 \times 10^{6}$ & $<8 \times 10^{6}$ & $<8 \times 10^{6}$ \\
\hline $\mathrm{U}$ & $<2 \times 10^{7}$ & $<2 \times 10^{7}$ & $<2 \times 10^{7}$ & $\mathrm{~N}_{<7 \times 10^{7}}$ & $\mathrm{~N}_{<8 \times 10^{7}}$ & $\mathrm{~N}_{<7 \times 10^{7}}$ \\
\hline I & ${ }^{\mathrm{B}} 1.3 \times 10^{5}$ & $3.2 \times 10^{5}$ & ${ }^{\mathrm{B}} 2.2 \pm 1.3 \times 10^{5}$ & ${ }^{\mathrm{B}} 5.5 \times 10^{5}$ & ${ }^{\mathrm{B}} 5.0 \times 10^{5}$ & $\mathrm{~B}_{5.3 \pm 0.38 \times 10^{5}}$ \\
\hline
\end{tabular}

"<"indicates a result that was less than the MDL. This is usually indicated by a "U" qualifier.

${ }^{\mathrm{B}}$ Concentration was greater than MDL but less than ten times MDL (ten times MDL is the EQL).

${ }^{\mathrm{N}}$ Matrix spike or matrix spike duplicate recoveries were outside the specified range (75 to125\%).

*This dissolution method is incompatible with determination of this analyte. 
The results given in Tables 5.1 were used along with the results to be discussed for the RCRA metals (Table 5.2) to determine an average glass waste form composition. On an oxide basis, this average composition would account for $99 \%$ (using only results above the EQLs) of the waste form. After adding titanium results from SRTC measurements ${ }^{4}$ the total oxide concentration was $100 \%$. Therefore, these analyses were successful at determining the concentration of all species present at concentrations of $0.5 \mathrm{wt} \%$ and above. These calculations have been included in the data package for these analyses.

\section{A.1.b. RCRA Metals}

Concentrations are given in Table 5.2 for the RCRA metals. These results showed that for the RCRA metals,

-For all analytes, at least one set of samples met all quality control requirements. -Chromium, nickel, and zinc were the only RCRA metals present above the EQLs. -Arsenic, barium, beryllium, mercury, and selenium concentrations met UTS limits.

Table 5.2. Concentrations of RCRA Metal Analytes Measured by ICP-ES and AA

\begin{tabular}{|c|c|c|c|c|c|c|}
\hline & \multicolumn{3}{|c|}{ Acid Dissolution Samples $(\mu \mathrm{g} / \mathrm{kg})$} & \multicolumn{3}{|c|}{ Peroxide Fusion Samples $(\mu \mathrm{g} / \mathrm{kg})$} \\
\hline & AA1 & AA2 & Average & FA1 & FA2 & Average \\
\hline Sb & $\mathrm{N}_{<1 \times 10^{5}}$ & $\mathrm{~N}_{<1 \times 10^{5}}$ & $\mathrm{~N}_{<1.4 \times 10^{5}}$ & $<7 \times 10^{5}$ & $<7 \times 10^{5}$ & $<7 \times 10^{5}$ \\
\hline As (AA) & $<2 \times 10^{3}$ & $<2 \times 10^{3}$ & $<2 \times 10^{3}$ & $<8 \times 10^{3}$ & $<8 \times 10^{3}$ & $<8 \times 10^{3}$ \\
\hline As (ES) & ${ }^{\mathrm{B}} 2.7 \times 10^{5}$ & ${ }^{B_{3}} 3.0 \times 10^{5}$ & ${ }^{\mathrm{B}} 2.9 \pm 0.17 \times 10^{5}$ & ${ }^{\mathrm{B}} 5.3 \times 10^{5}$ & $<3 \times 10^{5}$ & ${ }^{\mathrm{B}} 5.3 \times 10^{5}$ \\
\hline Ba & ${ }^{\mathrm{B}} 5.7 \times 10^{4}$ & $9.7 \times 10^{4}$ & ${ }^{B} 7.7 \pm 2.8 \times 10^{4}$ & ${ }^{\mathrm{B}} 4.9 \times 10^{4}$ & $<4 \times 10^{4}$ & ${ }^{\mathrm{B}} 4.9 \times 10^{4}$ \\
\hline Be & $<2 \times 10^{3}$ & $<2 \times 10^{3}$ & $<2 \times 10^{3}$ & $<8 \times 10^{3}$ & $<8 \times 10^{3}$ & $<8 \times 10^{3}$ \\
\hline Cd & ${ }^{\mathrm{B}} 1.6 \times 10^{4}$ & ${ }^{\mathrm{B}} 1.1 \times 10^{4}$ & ${ }^{\mathrm{B}} 1.3 \pm 0.39 \times 10^{4}$ & ${ }^{\mathrm{B}} 3.7 \times 10^{4}$ & ${ }^{B} 4.4 \times 10^{4}$ & ${ }^{\mathrm{B}} 4.1 \pm 0.46 \times 10^{4}$ \\
\hline $\mathbf{C r}$ & $5.59 \times 10^{5}$ & $5.50 \times 10^{5}$ & $5.55 \pm 0.063 \times 10^{5}$ & ${ }^{\mathrm{B}} 6.1 \times 10^{5}$ & ${ }^{\mathrm{B}} 5.9 \times 10^{5}$ & ${ }^{\mathrm{B}} 6.0 \pm 0.16 \times 10^{5}$ \\
\hline $\mathbf{P b}$ & $<2 \times 10^{5}$ & $<2 \times 10^{5}$ & $<2 \times 10^{5}$ & ${ }^{\mathrm{B}} 8.2 \times 10^{5}$ & $<8 \times 10^{5}$ & ${ }^{\mathrm{B}} 8.2 \times 10^{5}$ \\
\hline Hg & $<20$ & $<20$ & $<20$ & * & * & $*$ \\
\hline $\mathbf{N i}$ & $2.54 \times 10^{5}$ & $2.66 \times 10^{5}$ & $2.60 \pm 0.090 \times 10^{5}$ & * & * & * \\
\hline Se (AA) & ${ }^{\mathrm{B}} 4.9 \times 10^{3}$ & ${ }^{\mathrm{B}} 4.2 \times 10^{3}$ & ${ }^{\mathrm{B}} 4.5 \pm 0.55 \times 10^{3}$ & $\mathrm{~N}_{<}<1 \times 10^{4}$ & $\mathrm{~N}^{\mathrm{N}}<1 \times 10^{4}$ & $\mathrm{~N}_{<}<1 \times 10^{4}$ \\
\hline Se (ES) & $<9 \times 10^{4}$ & $<9 \times 10^{4}$ & $<9 \times 10^{4}$ & $<4 \times 10^{5}$ & $<4 \times 10^{5}$ & $<4 \times 10^{5}$ \\
\hline $\mathbf{A g}$ & ${ }^{\mathrm{B}} 1.18 \times 10^{5}$ & ${ }^{\mathrm{B}} 1.15 \times 10^{5}$ & ${ }^{\mathrm{B}} 1.15 \pm 0.044 \times 10^{5}$ & ${ }^{\mathrm{B}} 1.7 \times 10^{5}$ & $<1 \times 10^{5}$ & ${ }^{B} 1.7 \times 10^{5}$ \\
\hline Tl & $<3 \times 10^{5}$ & $<3 \times 10^{5}$ & $<3 \times 10^{5}$ & $<1 \times 10^{6}$ & $<1 \times 10^{6}$ & $<1 \times 10^{6}$ \\
\hline $\mathbf{V}$ & ${ }^{\mathrm{B}} 4.0 \times 10^{4}$ & ${ }^{\mathrm{B}} 3.1 \times 10^{4}$ & ${ }^{B} 3.5 \pm 0.65 \times 10^{4}$ & ${ }^{\mathrm{B}} 6.0 \times 10^{4}$ & ${ }^{\mathrm{B}} 7.2 \times 10^{4}$ & ${ }^{\mathrm{B}} 6.6 \pm 0.85 \times 10^{4}$ \\
\hline $\mathbf{Z n}$ & $2.38 \times 10^{7}$ & $2.35 \times 10^{7}$ & $2.36 \pm 0.017 \times 10^{7}$ & $2.43 \times 10^{7}$ & $2.33 \times 10^{7}$ & $2.38 \pm 0.068 \times 10^{7}$ \\
\hline
\end{tabular}

"<"indicates a result that was less than the MDL. This is usually indicated by a "U" qualifier. ${ }^{\mathrm{B}}$ Concentration was greater than MDL but less than ten times MDL (ten times MDL is the EQL). ${ }^{\mathrm{N}}$ Matrix spike or matrix spike duplicate recoveries were outside the specified range (75 to125\%). *This dissolution method is incompatible with determination of this analyte. 


\section{WSRC-TR-2001-00584, Revision 0}

SRT-RPP-2001-00220, Revision 0

Chromium, nickel, and zinc were the only RCRA metals present in the glass waste form at concentrations greater than the EQLs. The acid and peroxide fusion results were similar chromium and zinc. Nickel could not be determined in the peroxide-fusion samples because nickel crucibles were used for the dissolution. For arsenic (as determined by AA), barium, beryllium, mercury, and selenium, the results given in Table 5.2 were low enough to show that the waste form would meet UTS requirements even if these elements were completely released during TCLP tests. Arsenic concentrations determined by ICP-ES were two orders of magnitude higher than the AA DLs. The ICP-ES arsenic results should be considered suspect because AA is generally considered to be the preferred method for this analyte and because similar results were seen for the process blank (which contained no arsenic).

\section{A.2 Comparison of Results to Target Composition and to SRTC Results}

In this section, averages from the acid-dissolved samples and the peroxide-fusion samples have been compared to similar results determined from analyses performed at SRTC on this glass waste form. Although the analytical methods were similar for the SRTC analyses, not all of the $\mathrm{SW}-846$ protocol was followed for these analyses. For analytes that were detected in one set of samples but not the other, the detected value was presented with the averages.

\section{A.2.a. Non-RCRA Metals}

Results from these USEPA SW-846 non-RCRA metal analyses are given in Table 5.3 along with analytical results measured at SRTC. All primary component (those present above $10^{6} \mu \mathrm{g} / \mathrm{kg}$ ) concentrations were similar for the analyses given in this report and for those reported previously by SRTC except for boron. Results in Table 5.3 also include a normalized target composition that was determined by adjusting the initial target (provided by VSL) by experimental parameters (actual amounts of glass formers and supernate, impurities in the glass formers, etc). These calculations have been discussed elsewhere. ${ }^{4}$ For manganese, acid results have been used because of unexpectedly high peroxide fusion values in both the USEPA SW-846 and the SRTC analyses.

\section{A.2.b. RCRA Metals}

Results from these USEPA SW-846 RCRA metal analyses are given in Table 5.4 along with analytical results measured at SRTC. Concentrations were similar for analyses given in this report and for SRTC analyses except cadmium values. Results in Table 5.4 also indicated measured concentrations were similar to target values for RCRA metals except chromium and nickel. These apparent deviations were most likely due to uncertainty associated with chromium determination in the glass and the nickel measurement used to develop the target composition. 
WSRC-TR-2001-00584, Revision 0

SRT-RPP-2001-00220, Revision 0

Table 5.3. Non-RCRA Metal Results Compared to SRTC Results and to the Target Composition

\begin{tabular}{|c|c|c|c|c|}
\hline & SW-846 Results $(\mu \mathrm{g} / \mathrm{kg})$ & $* * *$ SRTC Results $(\mu \mathrm{g} / \mathrm{kg})$ & \multicolumn{2}{|c|}{ Target $(\mu \mathrm{g} / \mathrm{kg})$} \\
\hline & & & *Initial & $* *$ Normalized \\
\hline Al & $2.7 \pm 0.23 \times 10^{7}$ & $2.8 \pm 0.37 \times 10^{7}$ & $3.3 \times 10^{7}$ & $3.5 \times 10^{7}$ \\
\hline $\mathbf{C a}$ & $4.0 \pm 0.52 \times 10^{7}$ & $3.9 \pm 0.42 \times 10^{7}$ & $4.6 \times 10^{7}$ & $4.7 \times 10^{7}$ \\
\hline Co & ${ }^{\mathrm{B}} 2.0 \times 10^{5}$ & $6.0 \times 10^{5}$ & $1.6 \times 10^{3}$ & $1.6 \times 10^{3}$ \\
\hline $\mathrm{Cu}$ & $\mathrm{B}_{2} .8 \pm 0.23 \times 10^{5}$ & $2.5 \pm 0.71 \times 10^{5}$ & $3.2 \times 10^{3}$ & $2.7 \times 10^{3}$ \\
\hline $\mathbf{F e}$ & $4.1 \pm 0.13 \times 10^{7}$ & $4.28 \pm 0.014 \times 10^{7}$ & $4.5 \times 10^{7}$ & $4.6 \times 10^{7}$ \\
\hline Mg & $8.1 \pm 0.42 \times 10^{6}$ & $8.3 \pm 0.35 \times 10^{6}$ & $9.2 \times 10^{6}$ & $9.4 \times 10^{6}$ \\
\hline Mn & $2.11 \pm 0.044 \times 10^{5}$ & $2.0 \times 10^{5}$ & $6.3 \times 10^{2}$ & $1.1 \times 10^{3}$ \\
\hline $\mathbf{K}$ & ${ }^{\mathrm{B}} 1.2 \times 10^{6}$ & $9.5 \pm 3.5 \times 10^{5}$ & $6.2 \times 10^{5}$ & $6.8 \times 10^{5}$ \\
\hline $\mathbf{N a}$ & $7.2 \times 10^{7}$ & $6.9 \times 10^{7}$ & $8.8 \times 10^{7}$ & $7.6 \times 10^{7}$ \\
\hline Mo & $<4 \times 10^{4}$ & - & $1.7 \times 10^{4}$ & $1.7 \times 10^{4}$ \\
\hline $\mathbf{L i}$ & $1.3 \times 10^{7}$ & $1.2 \pm 0.13 \times 10^{7}$ & $1.3 \times 10^{7}$ & $1.3 \times 10^{7}$ \\
\hline $\mathbf{P}$ & ${ }^{\mathrm{B}} 6.5 \pm 0.65 \times 10^{5}$ & $8.5 \pm 2.1 \times 10^{5}$ & - & - \\
\hline $\mathbf{Z r}$ & $2.6 \pm 0.17 \times 10^{7}$ & $2.3 \pm 0.18 \times 10^{7}$ & $2.2 \times 10^{7}$ & $2.3 \times 10^{7}$ \\
\hline $\mathbf{S}$ & $1.42 \pm 0.081 \times 10^{6}$ & - & - & - \\
\hline Sn & $<1 \times 10^{5}$ & $2.0 \times 10^{5}$ & $1.2 \times 10^{4}$ & $1.0 \times 10^{4}$ \\
\hline $\mathbf{S i}$ & $2.3 \pm 0.12 \times 10^{8}$ & $2.1 \pm 0.20 \times 10^{8}$ & $2.2 \times 10^{8}$ & $2.2 \times 10^{8}$ \\
\hline B & $3.7 \times 10^{7}$ & $3.0 \times 10^{7}$ & $3.2 \times 10^{7}$ & $3.2 \times 10^{7}$ \\
\hline $\mathbf{W}$ & $<3 \times 10^{5}$ & - & - & - \\
\hline Ta & $\mathrm{BN}_{1.1 \times 10^{6}}$ & - & - & - \\
\hline $\mathbf{B i}$ & $<6 \times 10^{5}$ & - & - & - \\
\hline $\mathbf{P t}$ & $<1 \times 10^{5}$ & - & - & - \\
\hline $\mathbf{Y}$ & $<6 \times 10^{4}$ & - & - & - \\
\hline $\mathbf{P d}$ & ${ }^{B} 9.2 \times 10^{6}$ & - & - & - \\
\hline $\mathbf{R h}$ & $<2 \times 10^{6}$ & - & - & - \\
\hline $\mathbf{U}$ & $<2 \times 10^{7}$ & - & - & - \\
\hline I & $\mathrm{B}_{3.7 \pm 2.2 \times 10^{5}}$ & - & - & - \\
\hline
\end{tabular}

"<"indicates a result that was less than the MDL. This is usually indicated by a "U" qualifier.

${ }^{\mathrm{B}}$ Concentration was greater than MDL but less than ten times MDL (ten times MDL is the EQL).

${ }^{\mathrm{N}}$ Matrix spike or matrix spike duplicate recoveries were outside specified range (75 to125\%). ${ }^{1}$

* These values were determined from the oxide composition specified by VSL.

**These values were adjusted to account for actual quantities of waste and glass formers used. ${ }^{5}$

***These results were from analysis of the same glass waste form performed at SRTC. ${ }^{5}$ 
Table 5.4. RCRA Metal Results Compared to SRTC Results and to the Target Composition

\begin{tabular}{|c||c|c|c|}
\hline & USEPA SW-846 Results $(\mu \mathrm{g} / \mathrm{kg})$ & $* *$ SRTC Results $(\mu \mathrm{g} / \mathrm{kg})$ & $*$ Target $(\mu \mathrm{g} / \mathrm{kg})$ \\
\hline $\mathbf{S b}$ & $<7 \times 10^{5}$ & - & - \\
\hline $\mathbf{A s}(\mathbf{E S})$ & ${ }^{\mathrm{B}} 2.9 \times 10^{5}$ & - & - \\
\hline $\mathbf{B a}$ & ${ }^{\mathrm{B}} 7.7 \times 10^{4}$ & $8.0 \times 10^{5}$ & - \\
\hline $\mathbf{B e}$ & $<2 \times 10^{3}$ & - & $1.7 \times 10^{4}$ \\
\hline $\mathbf{C d}$ & ${ }^{\mathrm{B}} 2.7 \pm 1.9 \times 10^{4}$ & $1.0 \times 10^{5}$ & $1.2 \times 10^{5}$ \\
\hline $\mathbf{C r}$ & ${ }^{\mathrm{B}} 5.8 \pm 0.34 \times 10^{5}$ & $6.0 \times 10^{5}$ & $4.0 \times 10^{4}$ \\
\hline $\mathbf{P b}$ & $<2 \times 10^{5}$ & $4.0 \pm 2.8 \times 10^{5}$ & $1.0 \times 10^{5}$ \\
\hline $\mathbf{N i}$ & $2.6 \times 10^{5}$ & $3.0 \times 10^{5}$ & - \\
\hline Se (ES) & $<9 \times 10^{4}$ & - & - \\
\hline $\mathbf{A g}$ & ${ }^{\mathrm{B}} 1.1 \times 10^{5}$ & - & - \\
\hline $\mathbf{T l}$ & $<3 \times 10^{5}$ & - & $2.4 \times 10^{7}$ \\
\hline $\mathbf{V}$ & ${ }^{\mathrm{B}} 5.1 \pm 2.2 \times 10^{4}$ & $1.0 \times 10^{5}$ & - \\
\hline $\mathbf{Z n}$ & $2.37 \pm 0.011 \times 10^{7}$ & $2.57 \pm 0.057 \times 10^{7}$ & - \\
\hline $\mathbf{H g}$ & $<20$ & - & - \\
\hline $\mathbf{A s}$ (AA) & $<2 \times 10^{3}$ & - & - \\
\hline Se (AA) & ${ }^{\mathrm{B}} 4.5 \times 10^{3}$ & - & $\mathrm{C}$ \\
\hline
\end{tabular}

"<"indicates a result that was less than the MDL. This is usually indicated by a "U" qualifier.

${ }^{\mathrm{B}}$ Concentration was greater than MDL but less than ten times MDL (EQL is ten times MDL).

${ }^{\mathrm{N}}$ Matrix spike or matrix spike duplicate recoveries outside the specified range (75 to125\%). ${ }^{1}$

* These results were determined from the oxide composition specified by VSL.

**These results were from analysis of the same glass waste form performed at SRTC. ${ }^{5}$

\section{A.3 Comparison of Results to Results from a Tank AN-102 Crucible-Scale Vitrification}

In this section, averages from the acid-dissolved samples and the peroxide fusion samples are compared to similar averages from a crucible-scale vitrification that was performed with this waste form. For analytes that were detected in one set of samples but not the other, the detected value was presented with the averages. In addition, for cases in which only one set of analyses met the recovery specifications, only that result was included with the averages.

\section{A.3.a. Non-RCRA Metals}

Results from these non-RCRA metal analyses have been given in Table 5.5 along with analytical results from a crucible-scale vitrification study that was performed at SRTC with the Tank AN102 sample. Results given in Table 5.5 showed the concentrations to be similar for all analytes present above $10^{6} \mu \mathrm{g} / \mathrm{kg}$. In fact, only cobalt and copper concentrations were significantly different, and these analytes were reported at concentrations less than the EQLs. 
WSRC-TR-2001-00584, Revision 0

SRT-RPP-2001-00220, Revision 0

Table 5.5. Non-RCRA Metal Results Compared to Results from a Crucible-Scale Vitrification

\begin{tabular}{|c|c|c|c|c|c|}
\hline & \multicolumn{2}{|c|}{ Melter Waste Form $(\mu \mathrm{g} / \mathbf{k g})$} & \multicolumn{3}{|c|}{ Crucible Waste Form $(\mu \mathrm{g} / \mathrm{kg})$} \\
\hline & SW-846 & *Target & SW-846 & **SRTC & *Target \\
\hline Al & $2.8 \pm 0.28 \times 10^{7}$ & $3.3 \times 10^{7}$ & $3.4 \times 10^{7}$ & $3.3 \pm 0.28 \times 10^{7}$ & $3.3 \times 10^{7}$ \\
\hline $\mathbf{C a}$ & $4.1 \pm 0.59 \times 10^{7}$ & $4.6 \times 10^{7}$ & $4.7 \pm 0.11 \times 10^{7}$ & $4.5 \pm 0.33 \times 10^{7}$ & $4.6 \times 10^{7}$ \\
\hline Co & ${ }^{B} 2.1 \times 10^{5}$ & $1.6 \times 10^{3}$ & ${ }^{B} 9.8 \times 10^{4}$ & - & $1.6 \times 10^{3}$ \\
\hline $\mathrm{Cu}$ & ${ }^{B} 2.9 \times 10^{5}$ & $3.2 \times 10^{3}$ & ${ }^{\mathrm{B}} 8.9 \pm 3.0 \times 10^{4}$ & $1.5 \pm 0.71 \times 10^{5}$ & $3.2 \times 10^{3}$ \\
\hline $\mathbf{F e}$ & $4.2 \pm 0.20 \times 10^{7}$ & $4.5 \times 10^{7}$ & $4.48 \pm 0.035 \times 10^{7}$ & $4.9 \pm 0.56 \times 10^{7}$ & $4.5 \times 10^{7}$ \\
\hline Mg & $8.3 \pm 0.56 \times 10^{6}$ & $9.2 \times 10^{6}$ & $9.25 \pm 0.071 \times 10^{6}$ & $9.8 \pm 0.42 \times 10^{6}$ & $9.1 \times 10^{6}$ \\
\hline Mn & $8.2 \pm 8.6 \times 10^{5}$ & $6.3 \times 10^{2}$ & $4.6 \pm 3.5 \times 10^{5}$ & $6.5 \pm 6.4 \times 10^{5}$ & $6.3 \times 10^{2}$ \\
\hline $\mathbf{N i}$ & $2.6 \times 10^{5}$ & $1.0 \times 10^{5}$ & ${ }^{\mathrm{B}} 1.9 \times 10^{5}$ & $2.0 \times 10^{5}$ & $9.7 \times 10^{4}$ \\
\hline $\mathbf{K}$ & ${ }^{\mathrm{B}} 1.2 \times 10^{6}$ & $6.2 \times 10^{5}$ & $\mathrm{~B}_{1.3 \pm 0.15 \times 10^{6}}$ & $1.1 \pm 0.57 \times 10^{6}$ & $7.5 \times 10^{5}$ \\
\hline $\mathrm{Na}$ & $7.3 \times 10^{7}$ & $8.8 \times 10^{7}$ & $9.1 \times 10^{7}$ & $9.0 \times 10^{7}$ & $8.8 \times 10^{7}$ \\
\hline $\mathbf{Z n}$ & $2.43 \pm 0.049 \times 10^{7}$ & $2.4 \times 10^{7}$ & $2.4 \times 10^{7}$ & $2.5 \pm 0.13 \times 10^{7}$ & $2.4 \times 10^{7}$ \\
\hline Mo & $<4 \times 10^{4}$ & $1.7 \times 10^{4}$ & $<6 \times 10^{4}$ & $1.0 \times 10^{5}$ & $1.7 \times 10^{4}$ \\
\hline $\mathbf{L i}$ & $1.3 \times 10^{7}$ & $1.3 \times 10^{7}$ & ${ }^{\mathrm{J}} 1.28 \pm 0.035 \times 10^{7}$ & $1.27 \pm 0.35 \times 10^{7}$ & $1.3 \times 10^{7}$ \\
\hline $\bar{P}$ & B $6.7 \pm 0.17 \times 10^{5}$ & - & ${ }^{\mathrm{B}} 5.65 \pm 0.071 \times 10^{5}$ & $9.5 \pm 0.71 \times 10^{5}$ & $5.6 \times 10^{5}$ \\
\hline $\mathbf{Z r}$ & $2.6 \pm 0.21 \times 10^{7}$ & $2.2 \times 10^{7}$ & $2.33 \pm 0.035 \times 10^{7}$ & $2.43 \pm 0.057 \times 10^{7}$ & $2.2 \times 10^{7}$ \\
\hline $\mathbf{S}$ & $1.5 \pm 0.11 \times 10^{6}$ & - & $1.80 \pm 0.071 \times 10^{6}$ & - & $1.4 \times 10^{6}$ \\
\hline Sn & $<1 \times 10^{5}$ & $1.2 \times 10^{4}$ & $<2 \times 10^{5}$ & $2.5 \pm 0.71 \times 10^{5}$ & $8.7 \times 10^{3}$ \\
\hline $\mathbf{S i}$ & $2.4 \pm 0.16 \times 10^{8}$ & $2.2 \times 10^{8}$ & $2.0 \pm 0.11 \times 10^{8}$ & $2.29 \pm 0.026 \times 10^{8}$ & $2.2 \times 10^{8}$ \\
\hline B & $3.8 \times 10^{7}$ & $3.2 \times 10^{7}$ & $3.0 \times 10^{7}$ & $3.4 \times 10^{7}$ & $3.1 \times 10^{7}$ \\
\hline $\mathbf{W}$ & $<3 \times 10^{5}$ & - & $\mathrm{J}^{\mathrm{J}}<3 \times 10^{5}$ & - & - \\
\hline Ta & $\mathrm{BN}_{1.1 \times 10^{6}}$ & - & $\mathrm{JB}^{\mathrm{JB}} 8.7 \pm 0.21 \times 10^{5}$ & - & - \\
\hline $\mathbf{B i}$ & $<6 \times 10^{5}$ & - & $<9 \times 10^{4}$ & - & - \\
\hline $\mathbf{P t}$ & $<1 \times 10^{5}$ & - & $\mathrm{JB}_{1.4 \times 10^{5}}$ & - & - \\
\hline $\mathbf{Y}$ & $<6 \times 10^{4}$ & - & $\mathrm{B}_{3.6 \pm 0.28 \times 10^{4}}$ & - & - \\
\hline Pd & ${ }^{\mathrm{B}} 9.5 \times 10^{6}$ & - & $1.08 \pm 0.035 \times 10^{7}$ & - & - \\
\hline $\mathbf{R h}$ & $<2 \times 10^{6}$ & - & ${ }^{\top}<2 \times 10^{6}$ & - & - \\
\hline $\mathbf{U}$ & $<2 \times 10^{7}$ & - & ${ }^{\mathrm{J}}<3 \times 10^{6}$ & - & - \\
\hline I & ${ }^{\mathrm{B}} 5.5 \times 10^{2}$ & - & $x^{2}$ & - & - \\
\hline
\end{tabular}

"<"indicates a result that was less than the MDL. This is usually indicated by a "U" qualifier.

${ }^{\mathrm{B}}$ Concentration was greater than MDL but less than ten times MDL (EQL is ten times MDL).

${ }^{\mathrm{N}}$ Matrix spike or matrix spike duplicate recoveries were outside specified range (75 to125\%). ${ }^{1}$

* These results were determined from the oxide composition specified by VSL.

**These results were from analysis of the same glass waste form performed at SRTC. ${ }^{5}$

5.A.3.b. RCRA Metals 
Results from these RCRA metal analyses are given in Table 5.6 along with analytical results from the SRTC crucible-scale vitrification study. Results given in Table 5.6 showed the concentrations to be similar for all RCRA metal analytes except barium and chromium. These apparent deviations were most likely due to uncertainties in determining these analytes at concentrations close to the detection limits. Although the ICP-ES values for arsenic were also high in the melter samples, AA results were thought to be more reliable for arsenic.

Table 5.6. RCRA Metal Results Compared to Results from a Crucible-Scale Vitrification

\begin{tabular}{|c|c|c|c|c|c|}
\hline & \multicolumn{2}{|c|}{ Melter Waste Form $(\mu \mathrm{g} / \mathrm{kg})$} & \multicolumn{3}{|c|}{ Crucible Waste Form $(\mu \mathrm{g} / \mathrm{kg})$} \\
\hline & SW-846 & *Target & SW-846 & $* *$ SRTC & *Target \\
\hline $\mathbf{S b}$ & $<7 \times 10^{5}$ & 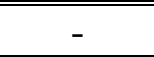 & $<8 \times 10^{4}$ & 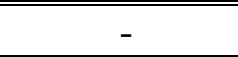 & - \\
\hline As (ES) & ${ }^{\mathrm{B}} 2.9 \times 10^{5}$ & - & $<2 \times 10^{3}$ & - & - \\
\hline Ba & $7.8 \times 10^{4}$ & - & $\mathrm{B}_{3.8 \times 10^{4}}$ & $<1 \times 10^{5}$ & - \\
\hline Be & $<2 \times 10^{3}$ & - & $<9 \times 10^{2}$ & - & - \\
\hline $\mathbf{C d}$ & $\mathrm{B}_{2.8 \pm 2.0 \times 10^{4}}$ & $1.7 \times 10^{4}$ & $\mathrm{~B}_{2.63 \pm 0.035 \times 10^{4}}$ & $1.0 \times 10^{5}$ & $1.6 \times 10^{4}$ \\
\hline $\mathrm{Cr}$ & ${ }^{\mathrm{B}} 5.9 \pm 0.44 \times 10^{5}$ & $1.2 \times 10^{5}$ & $2.3 \pm 0.11 \times 10^{5}$ & $7.5 \pm 6.4 \times 10^{5}$ & $1.1 \times 10^{5}$ \\
\hline $\mathbf{P b}$ & $<2 \times 10^{5}$ & $4.0 \times 10^{4}$ & ${ }^{\mathrm{B}} 1.7 \pm 0.49 \times 10^{5}$ & $2.0 \times 10^{5}$ & $3.5 \times 10^{4}$ \\
\hline $\mathbf{N i}$ & $2.6 \times 10^{5}$ & $1.0 \times 10^{5}$ & ${ }^{\mathrm{B}} 1.9 \times 10^{5}$ & $2.0 \times 10^{5}$ & $9.7 \times 10^{4}$ \\
\hline Se (ES) & $<9 \times 10^{4}$ & - & ${ }^{\mathrm{R}}<5 \times 10^{3}$ & - & - \\
\hline Ag & ${ }^{\mathrm{B}} 1.2 \times 10^{5}$ & - & ${ }^{\mathrm{J}} 1.3 \pm 0.28 \times 10^{5}$ & - & - \\
\hline $\mathbf{T l}$ & $<3 \times 10^{5}$ & - & $<2 \times 10^{5}$ & - & - \\
\hline $\mathbf{V}$ & ${ }^{\mathrm{B}} 5.2 \pm 2.3 \times 10^{4}$ & - & ${ }^{\mathrm{B}} 6.9 \pm 1.2 \times 10^{4}$ & $1.0 \times 10^{5}$ & - \\
\hline Zn & $2.43 \pm 0.049 \times 10^{7}$ & $2.4 \times 10^{7}$ & $2.4 \times 10^{7}$ & $2.5 \pm 0.13 \times 10^{7}$ & $2.4 \times 10^{7}$ \\
\hline $\mathrm{Hg}$ & $<20$ & - & $4.4 \times 10^{2}$ & - & - \\
\hline As (AA) & $<2 \times 10^{3}$ & - & - & - & - \\
\hline $\operatorname{Se}(\mathbf{A A})$ & ${ }^{\mathrm{B}} 4.5 \times 10^{3}$ & - & - & - & - \\
\hline
\end{tabular}

"<"indicates a result that was less than the MDL. This is usually indicated by a "U" qualifier.

${ }^{\mathrm{B}}$ Concentration was greater than MDL but less than ten times MDL (EQL is ten times MDL).

${ }^{\mathrm{N}}$ Matrix spike or matrix spike duplicate recoveries were outside specified range (75 to125\%). ${ }^{1}$

* These results were determined from the oxide composition specified by VSL.

**These results were from analysis of the same glass waste form performed at SRTC. ${ }^{5}$

\section{A.4 Toxicity Characteristic Leaching Procedure (TCLP)}

Concentrations have been given in Table 5.7 for the TCLP extract results. These results show

-The analytes given in Table 5.7 met the Universal Treatment Standard (UTS) limits.

-Barium concentrations were elevated although still an order of magnitude below the UTS limits.

-For the standard, some analyte results were up to $40 \%$ below the performance limits.

-The nonradioactive simulant and low-activity test reference material glass did not act as blanks for TCLP tests. 
All analytes given in Table 5.7 met UTS specifications. For the standard, some analytes were below the performance limits. TCLP does not mention use of such standards. One approach for implementing the standard is to correct appropriate sample results by the ratio of the standard's certified value to the measured standard value. These results have been included in parentheses in Table 5.7 for analytes that fell outside the standard performance limits. Even after application of the correction, all results were more than an order of magnitude below the UTS limits.

Table 5.7. Concentration of RCRA Metals in TCLP Extract

\begin{tabular}{|c|c|c|c|c|c|c|}
\hline & UTS Limit $(\mu \mathrm{g} / \mathrm{L})$ & Sample $(\mu \mathrm{g} / \mathrm{L})$ & \multicolumn{2}{|c|}{ Blanks $(\mu \mathrm{g} / \mathrm{L})$} & \multicolumn{2}{|c|}{ Standard $(\mu \mathrm{g} / \mathrm{L})$} \\
\hline & & TA1 & Simulant & LRM & Measured & Target \\
\hline $\mathbf{S b}$ & $1.15 \times 10^{3}$ & $<39(<80)$ & $<39$ & $<39$ & $1.0 \times 10^{3}$ & $1.3-2.8 \times 10^{3}$ \\
\hline $\operatorname{As}(\mathrm{ES})$ & $5 \times 10^{3}$ & $<19(<41)$ & ${ }^{\mathrm{B}} 30$ & ${ }^{\mathrm{B}} 24$ & $9.0 \times 10^{2}$ & $1.3-2.6 \times 10^{3}$ \\
\hline $\mathbf{A s}(\mathbf{A A})$ & $5 \times 10^{3}$ & $\mathrm{~N}_{<0.5(<2)}$ & $\mathrm{N}_{<0.5}$ & $\mathrm{~N}_{<0.5}$ & $\mathrm{~N}_{5.6 \times 10^{2}}$ & $1.3-2.6 \times 10^{3}$ \\
\hline $\mathbf{B a}$ & $2.1 \times 10^{4}$ & ${ }^{\mathrm{U}} 1.7 \times 10^{3}$ & $\mathrm{U}_{1.7 \times 10^{3}}$ & $\mathrm{U}_{1.3 \times 10^{3}}$ & $9.9 \times 10^{3}$ & $8.5-15 \times 10^{3}$ \\
\hline Be & $1.22 \times 10^{3}$ & $<0.4$ & $<0.4$ & $<0.4$ & $5.7 \times 10^{2}$ & $3.7-12 \times 10^{2}$ \\
\hline Cd & $1.1 \times 10^{2}$ & $\mathrm{~B}_{2.8}$ & $<2$ & 48 & $1.2 \times 10^{3}$ & $9.9-20 \times 10^{2}$ \\
\hline $\mathrm{Cr}$ & $6 \times 10^{2}$ & $<9(<22)$ & $<9$ & ${ }^{\mathrm{B}} 13$ & $4.8 \times 10^{2}$ & $6.5-17 \times 10^{2}$ \\
\hline $\mathbf{P b}$ & $7.5 \times 10^{2}$ & $<42$ & $<42$ & $<42$ & $1.2 \times 10^{3}$ & $4.3-28 \times 10^{2}$ \\
\hline $\mathrm{Ni}$ & $1.1 \times 10^{4}$ & ${ }^{\mathrm{B}} 14$ & ${ }^{\mathrm{B}} 13$ & ${ }^{\mathrm{B}} 19$ & $7.5 \times 10^{3}$ & $6.3-11 \times 10^{3}$ \\
\hline $\operatorname{Se}(\mathrm{ES})$ & $5.7 \times 10^{3}$ & $<24(<48)$ & $<24$ & $<24$ & $3.3 \times 10^{2}$ & $4.6-8.7 \times 10^{2}$ \\
\hline $\operatorname{Se}(\mathbf{A A})$ & $5.7 \times 10^{3}$ & ${ }^{\mathrm{B}} 1.0(<2.4)$ & $<0.8$ & $\begin{array}{l}\mathrm{B} 1.1 \\
\end{array}$ & $2.8 \times 10^{2}$ & $4.6-8.7 \times 10^{2}$ \\
\hline Ag & $1.4 \times 10^{2}$ & $<8$ & $<8$ & $<8$ & $5.7 \times 10^{2}$ & $5.1-11 \times 10^{2}$ \\
\hline $\mathbf{T l}$ & $2 \times 10^{2}$ & $<81$ & $<81$ & $<81$ & $<81$ & - \\
\hline $\mathrm{Hg}$ & 25 & $<0.1$ & $<0.1$ & $<0.1$ & 25 & $14-52$ \\
\hline
\end{tabular}

"<"indicates a result that was less than the MDL. This is usually indicated by a "U" qualifier.

"( )"Parentheses indicate the sample result after correcting for low standard results.

${ }^{\mathrm{B}}$ Concentration was greater than MDL but less than ten times MDL (EQL is ten times MDL).

${ }^{\mathrm{N}}$ Matrix spike or matrix spike duplicate recoveries were outside specified range (75 to125\%). ${ }^{1}$

${ }^{\mathrm{U}}$ Barium was regarded as not detected in data validation because of method blank contamination.

\section{B Inorganic Analytes Measured as Ionic Species}

Results for the target inorganic analytes that were determined as ionic species are given in Table 5.8. Of these, none were detected above the EQLs. Sulfate, ammonia, and total halide results were above the MDLs. For ammonia, similar concentrations were measured in the process and field blanks. Total halide (TX) results were significantly lower than the target value. This would be consistent with volatile species losses in vitrification. As shown in Table 5.9, these results were similar to results from related Tank AN-102 glass waste form characterizations. 
WSRC-TR-2001-00584, Revision 0

SRT-RPP-2001-00220, Revision 0

Table 5.8. Concentration of Target Inorganic Analytes Measured as Ionic Species

\begin{tabular}{|c|c|c|c|c|c|c|}
\hline & \multicolumn{4}{|c|}{ Samples (mg/kg) } & \multicolumn{2}{|c|}{ Standards (mg/kg) } \\
\hline & WA1 & WA2 & Average & *Target & Measured & $* *$ Actual \\
\hline $\mathbf{F}$ & $<5 \times 10^{3}$ & $<5 \times 10^{3}$ & $<5 \times 10^{3}$ & $5.3 \times 10^{2}$ & ${ }^{\mathrm{B}} 8.5 \pm 0.56 \times 10^{3}$ & $8.6 \pm 0.35 \times 10^{3}$ \\
\hline $\mathbf{B r}$ & $\mathrm{N}_{<5} \times 10^{3}$ & $\mathrm{~N}_{<5}<5 \times 10^{3}$ & $\mathrm{~N}_{<5} \times 10^{3}$ & - & ${ }^{\mathrm{N}}<5 \times 10^{3}$ & - \\
\hline $\mathrm{NO}_{3}$ & $<5 \times 10^{3}$ & $<5 \times 10^{3}$ & $<5 \times 10^{3}$ & - & $<5 \times 10^{3}$ & - \\
\hline $\mathrm{NO}_{2}$ & $<5 \times 10^{3}$ & $<5 \times 10^{3}$ & $<5 \times 10^{3}$ & - & $<5 \times 10^{3}$ & - \\
\hline $\mathrm{PO}_{4}$ & $\mathrm{~N}_{<5 \times 10^{3}}$ & $\mathrm{~N}_{<5 \times 10^{3}}$ & $\mathrm{~N}_{<5} \times 10^{3}$ & $1.1 \times 10^{3}$ & ${ }^{\mathrm{BN}} 6.3 \pm 0.43 \times 10^{3}$ & - \\
\hline $\mathrm{SO}_{4}$ & ${ }^{\mathrm{B}} 5.29 \times 10^{3}$ & ${ }^{\mathrm{B}} 5.36 \times 10^{3}$ & ${ }^{B} 5.32 \pm 0.045 \times 10^{3}$ & $3.8 \times 10^{3}$ & ${ }^{\mathrm{J}} 4.1 \pm 0.19 \times 10^{3}$ & $3.6 \pm 0.59 \times 10^{3}$ \\
\hline $\mathrm{Cl}$ & ${ }^{\mathrm{N}}<5 \times 10^{3}$ & $\mathrm{~N}_{<5 \times 10^{3}}$ & $\mathrm{~N}_{<5 \mathrm{x} 10^{3}}$ & $9.3 \times 10^{2}$ & $\mathrm{~N}_{<5} \times 10^{3}$ & $\begin{array}{llll}- \\
\end{array}$ \\
\hline & BA1 & BA2 & Average & *Target & - & - \\
\hline $\mathrm{NH}_{3}$ & ${ }^{\mathrm{B}} 1.2$ & ${ }^{\mathrm{B}} 0.90$ & ${ }^{\mathrm{B}} 1.1 \pm 0.23$ & 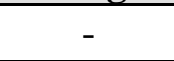 & - & - \\
\hline $\mathbf{C N}$ & $<0.05$ & $<0.05$ & $<0.05$ & - & - & - \\
\hline TX & ${ }^{\mathrm{B}} 1.6 \times 10^{2}$ & ${ }^{\mathrm{B}} 1.2 \times 10^{2}$ & ${ }^{\mathrm{B}} 1.4 \pm 0.30 \times 10^{2}$ & $1.5 \times 10^{3}$ & - & - \\
\hline
\end{tabular}

"<"indicates a result that was less than the MDL. This is usually indicated by a "U" qualifier.

${ }^{\mathrm{B}}$ Concentration was greater than MDL but less than ten times MDL (EQL is ten times MDL).

${ }^{\mathrm{J}}$ Analyte concentration was estimated because a peak was apparent but was below the MDL.

* These results were determined from the oxide composition specified by VSL.

**These results were from an interlaboratory study of the low-activity test reference material glass standard. ${ }^{5}$

${ }^{\mathrm{N}}$ Matrix spike or matrix spike duplicate recoveries were outside the specified range (75 to125\%).

Table 5.9. Comparison of Target Inorganic Analytes Measured as Ionic Species

\begin{tabular}{|c|c|c|c|c|c|c|}
\hline & \multicolumn{2}{|c|}{ Melter Waste Form (mg/kg) } & \multicolumn{3}{c|}{ Crucible Waste Form (mg/kg) } \\
\hline & $\mathbf{S W - 8 4 6}$ & $* *$ SRTC & $*$ Target & SW-846 & $* *$ SRTC & $*$ Target \\
\hline F & $<5 \times 10^{3}$ & - & $5.3 \times 10^{2}$ & ${ }^{3}<79$ & - & $6.0 \times 10^{2}$ \\
\hline PO $_{4}$ & $<5 \times 10^{3}$ & $1.4 \pm 0.47 \times 10^{3}$ & $1.1 \times 10^{3}$ & $1.8 \pm 0.11 \times 10^{3}$ & $1.6 \pm 0.31 \times 10^{3}$ & $1.7 \times 10^{3}$ \\
\hline SO $_{\mathbf{4}}$ & ${ }^{8} 5.32 \pm 0.045 \times 10^{3}$ & - & $3.8 \times 10^{3}$ & $3.44 \pm 0.029 \times 10^{3}$ & - & $3.6 \times 10^{3}$ \\
\hline $\mathbf{C l}$ & $<5 \times 10^{3}$ & - & $9.3 \times 10^{2}$ & ${ }^{\mathrm{J}}<78$ & - & $1.2 \times 10^{3}$ \\
\hline TX & ${ }^{\mathrm{B}} 1.4 \pm 0.30 \times 10^{2}$ & - & $1.5 \times 10^{3}$ & $76 \pm 33$ & - & $1.8 \times 10^{3}$ \\
\hline
\end{tabular}

"<"indicates a result that was less than the MDL. This is usually indicated by a "U" qualifier.

${ }^{\mathrm{B}}$ Concentration was greater than MDL but less than ten times MDL (EQL is ten times MDL).

${ }^{J}$ Analyte concentration was estimated because of apparent sodium interference.

* These results were determined from the oxide composition specified by VSL.

**These results were determined from the elemental composition determined at SRTC. 


\section{WSRC-TR-2001-00584, Revision 0 \\ SRT-RPP-2001-00220, Revision 0}

\section{C Radionuclide Analyses}

Results from radionuclide analyses have been given in this section. Results were in line with expected values, except curium-242 values were higher than anticipated. Results have been included in this section for all samples (two acid dissolutions and two peroxide fusions). In addition, averages have been included. For analytes not detected, the lower of the two "nondetects" have been given as the "average". For analytes detected in only one duplicate, only the detected value has been given as the "average".

\section{C.1 Measured Composition}

The concentrations of target radionuclides are given in Table 5.10. These results showed

-The total beta radioactivity in the Tank AN-102 waste form was less than $1.81 \times 10^{9} \mathrm{pCiVkg}$. -The total alpha radioactivity in the Tank AN-102 waste form was less than $2.47 \times 10^{7} \mathrm{pCiVkg}$. -Most beta radioactivity in the waste form was due to strontium-90.

-Most alpha radioactivity in the waste form was due to americium-241 and curium-244.

With the exception of the total alpha measurements, all primary radionuclide (radionuclides present at $10^{5} \mathrm{pCi} / \mathrm{kg}$ or higher) results were similar for the acid-dissolved samples and for the sodium peroxide fusion samples. The peroxide fusion sample total alpha results were approximately $25 \%$ higher than the results from the acid samples. The cause of this discrepancy was not apparent, especially since the precision within each pair of samples was very good. SRTC results are significantly lower than either of these results.

During data validation, some or all results for six radionuclides were rejected. The most significant was rejection of carbon-14 results due to interferences. Data validation of neptunium values indicated the results met all QA and QC requirements. Neptunium-243 nondetect values in Table 5.10 were higher than MDAs. These results were from analysis of samples spiked with a plutonium-242 tracer. Spectra from samples with and without the tracer showed no detectable neptunium-243. The difference between the MDA and sample results was due to tracer "taildown".

The curium-242 results given in Table 5.10 were unexpected. Because of its short halflife (163 days), curium-242 was not expected to be measurable. A review of the four spectra showed a small but well-shaped peak at the appropriate energy, $6.11 \mathrm{MeV}$. The only other radionuclide that would produce a peak at this energy would be californium-252 (6.12 MeV). Rough calculations showed that assuming this to be curium-242, the ratio of curium-244 to curium-242 ( 200) would be possible for waste up to 20 years old. 
WSRC-TR-2001-00584, Revision 0

SRT-RPP-2001-00220, Revision 0

Table 5.10. Concentration of Target Radionuclides

\begin{tabular}{|c|c|c|c|c|c|c|}
\hline & \multicolumn{3}{|c|}{ Acid Dissolution Samples (pCi/kg) } & \multicolumn{3}{|c|}{ Peroxide Fusion Samples (pCi/kg) } \\
\hline & AA1 & AA2 & Average & FA1 & FA2 & Average \\
\hline Alpha & $1.88 \times 10^{7}$ & $1.83 \times 10^{7}$ & $1.85 \pm 0.039 \times 10^{7}$ & $2.37 \times 10^{7}$ & $2.39 \times 10^{7}$ & $2.38 \pm 0.14 \times 10^{7}$ \\
\hline Beta & $1.54 \times 10^{9}$ & $1.65 \times 10^{9}$ & $1.59 \pm 0.078 \times 10^{9}$ & $1.75 \times 10^{9}$ & $1.75 \times 10^{9}$ & $1.75 \times 10^{9}$ \\
\hline Se-79 & $<7 \times 10^{6}$ & $<6 \times 10^{6}$ & $<6 \times 10^{6}$ & $<6 \times 10^{6}$ & $\mathrm{R}_{<2 \times 10^{7}}$ & $<6 \times 10^{6}$ \\
\hline $\mathrm{Np}-237$ & ${ }^{\mathrm{U}_{5}} .1 \times 10^{4}$ & $\mathrm{U}_{3.8 \times 10^{3}}$ & $\mathrm{U}_{2.7 \pm 3.3 \times 10^{4}}$ & ${ }^{\mathrm{U}} 8.7 \times 10^{3}$ & $\mathrm{U}_{1.3 \times 10^{4}}$ & $\mathrm{U}_{1.1 \pm 0.32 \times 10^{4}}$ \\
\hline $\mathrm{Pu} 239 / 240$ & $5.3 \times 10^{5}$ & $5.4 \times 10^{5}$ & $5.3 \pm 0.066 \times 10^{5}$ & $5.5 \times 10^{5}$ & $5.8 \times 10^{5}$ & $5.6 \pm 0.27 \times 10^{5}$ \\
\hline $\mathrm{Pu}-238$ & $4.3 \times 10^{5}$ & $4.7 \times 10^{5}$ & $4.5 \pm 0.28 \times 10^{5}$ & $4.71 \times 10^{5}$ & $4.67 \times 10^{5}$ & $4.69 \pm 0.030 \times 10^{5}$ \\
\hline $\mathrm{Pu}-241$ & $1.37 \times 10^{6}$ & $1.28 \times 10^{6}$ & $1.33 \pm 0.061 \times 10^{6}$ & $1.2 \times 10^{6}$ & $1.3 \times 10^{6}$ & $1.3 \pm 0.10 \times 10^{6}$ \\
\hline Am-241 & $8.4 \times 10^{6}$ & $8.2 \times 10^{6}$ & $8.3 \pm 0.16 \times 10^{6}$ & $8.6 \times 10^{6}$ & $8.1 \times 10^{6}$ & $8.4 \pm 0.39 \times 10^{6}$ \\
\hline *Am-241 & $8.3 \times 10^{6}$ & $9.1 \times 10^{6}$ & $8.7 \pm 0.56 \times 10^{6}$ & $0.99 \times 10^{7}$ & $1.10 \times 10^{7}$ & $1.05 \pm 0.077 \times 10^{7}$ \\
\hline $\mathrm{Cm}-244$ & $5.77 \times 10^{6}$ & $5.72 \times 10^{6}$ & $5.74 \pm 0.039 \times 10^{6}$ & $5.9 \times 10^{6}$ & $5.8 \times 10^{6}$ & $5.8 \pm 0.11 \times 10^{6}$ \\
\hline $\mathrm{Cm}-242$ & $2.9 \times 10^{4}$ & $4.6 \times 10^{4}$ & $3.8 \pm 1.2 \times 10^{4}$ & $4.3 \times 10^{4}$ & $1.2 \times 10^{4}$ & $2.7 \pm 2.2 \times 10^{4}$ \\
\hline U-234 & $\mathrm{U}_{2.8 \times 10^{4}}$ & $\mathrm{U}_{4.3 \times 10^{4}}$ & $\mathrm{U}_{3.6 \pm 1.0 \times 10^{4}}$ & $6.7 \times 10^{4}$ & $9.8 \times 10^{4}$ & $8.2 \pm 2.2 \times 10^{4}$ \\
\hline U-235 & ${ }^{\mathrm{J}} 2.4 \times 10^{3}$ & $\mathrm{~J}_{2} .1 \times 10^{3}$ & $\mathrm{~J}_{2} 2.2 \pm 0.27 \times 10^{3}$ & $\mathrm{R}_{4.4 \times 10^{3}}$ & ${ }^{\mathrm{J}} 6.4 \times 10^{3}$ & ${ }^{\mathrm{J}} 6.4 \times 10^{3}$ \\
\hline $\mathrm{U}-236$ & $\mathrm{R} 1.2 \times 10^{3}$ & $<9 \times 10^{2}$ & $<9 \times 10^{2}$ & $\mathrm{R} 1.3 \times 10^{3}$ & $\mathrm{R} 1.5 \times 10^{3}$ & $\mathrm{R}_{1.4 \pm 0.19 \times 10^{3}}$ \\
\hline $\mathrm{U}-238$ & $3.9 \times 10^{3}$ & $4.9 \times 10^{3}$ & $4.4 \pm 0.77 \times 10^{3}$ & $4.6 \times 10^{3}$ & $6.4 \times 10^{3}$ & $5.5 \pm 1.3 \times 10^{3}$ \\
\hline I-129 & $\mathrm{U}_{1.7 \times 10^{6}}$ & $\mathrm{U}_{7.6 \times 10^{5}}$ & $\mathrm{U}_{1.2 \pm 0.69 \times 10^{6}}$ & $\mathrm{U}_{1.8 \times 10^{6}}$ & $\mathrm{U}_{4.2 \times 10^{5}}$ & $\mathrm{U}_{1.1 \pm 0.97 \times 10^{6}}$ \\
\hline Tc-99 & $5.79 \times 10^{6}$ & $5.83 \times 10^{6}$ & $5.81 \pm 0.029 \times 10^{6}$ & $6.1 \times 10^{6}$ & $5.6 \times 10^{6}$ & $5.8 \pm 0.33 \times 10^{6}$ \\
\hline Sr-90 & $7.5 \times 10^{8}$ & $7.6 \times 10^{8}$ & $7.5 \pm 0.11 \times 10^{8}$ & $8.5 \times 10^{8}$ & $9.3 \times 10^{8}$ & $8.9 \pm 0.53 \times 10^{8}$ \\
\hline Co-60 & $2.0 \times 10^{7}$ & $1.8 \times 10^{7}$ & $1.9 \pm 0.12 \times 10^{7}$ & $2.0 \times 10^{7}$ & $2.1 \times 10^{7}$ & $2.1 \pm 0.11 \times 10^{7}$ \\
\hline $\mathrm{Nb}-94$ & $1.9 \times 10^{6}$ & $\mathrm{R}_{<2 \times 10^{5}}$ & $1.9 \times 10^{6}$ & $<3 \times 10^{5}$ & $<2 \times 10^{5}$ & $<2 \times 10^{5}$ \\
\hline $\mathrm{Ru}-103$ & $<2 \times 10^{5}$ & $<1 \times 10^{5}$ & $<1 \times 10^{5}$ & $<2 \times 10^{5}$ & $<1 \times 10^{5}$ & $<1 \times 10^{5}$ \\
\hline Ru-106 & $<1 \times 10^{6}$ & $<1 \times 10^{6}$ & $<1 \times 10^{6}$ & $<2 \times 10^{6}$ & $<1 \times 10^{6}$ & $<1 \times 10^{6}$ \\
\hline Cs-134 & $<1 \times 10^{5}$ & $<1 \times 10^{5}$ & $<1 \times 10^{5}$ & $<2 \times 10^{5}$ & $<1 \times 10^{5}$ & $<1 \times 10^{5}$ \\
\hline Cs-137 & $2.3 \times 10^{7}$ & $2.2 \times 10^{7}$ & $2.2 \pm 0.11 \times 10^{7}$ & $2.2 \times 10^{7}$ & $2.6 \times 10^{7}$ & $2.4 \pm 0.26 \times 10^{7}$ \\
\hline Eu-152 & $\mathrm{R}_{<4 \times 10^{5}}$ & $3.2 \times 10^{5}$ & $3.2 \times 10^{5}$ & $<5 \times 10^{5}$ & $<4 \times 10^{5}$ & $<4 \times 10^{5}$ \\
\hline Eu-154 & $1.89 \times 10^{7}$ & $1.80 \times 10^{7}$ & $1.85 \pm 0.064 \times 10^{7}$ & $2.13 \times 10^{7}$ & $2.21 \times 10^{7}$ & $2.17 \pm 0.059 \times 10^{7}$ \\
\hline Eu-155 & $1.05 \times 10^{7}$ & $1.01 \times 10^{7}$ & $1.03 \pm 0.025 \times 10^{7}$ & $1.14 \times 10^{7}$ & $1.23 \times 10^{7}$ & $1.19 \pm 0.067 \times 10^{7}$ \\
\hline Ra-226 & $<3 \times 10^{5}$ & $<2 \times 10^{5}$ & $<2 \times 10^{5}$ & $<3 \times 10^{5}$ & $<2 \times 10^{5}$ & $<2 \times 10^{5}$ \\
\hline C-14 & $\mathrm{R}_{<1 \times 10^{6}}$ & $\mathrm{R}_{<1 \times 10^{6}}$ & $\mathrm{R}_{<1 \times 10^{6}}$ & $\mathrm{R}_{2.9 \times 10^{6}}$ & $\mathrm{R}_{<1 \times 10^{6}}$ & $\mathrm{R}_{2.9 \times 10^{6}}$ \\
\hline $\mathrm{H}-3$ & $<2 \times 10^{6}$ & $<2 \times 10^{6}$ & $<2 \times 10^{6}$ & $<2 \times 10^{6}$ & $<2 \times 10^{6}$ & $<2 \times 10^{6}$ \\
\hline
\end{tabular}

"<"indicates a result that was less than the MDA. This is usually indicated by a "U" qualifier. ${ }^{J}$ Analyte regarded as detected, but validation showed its reported concentration to be uncertain. ${ }^{\mathrm{R}}$ The analyte result was determined to be unusable during data validation.

UThe analyte was regarded as not detected during data validation.

*These americium-241 results were determined from the gamma pulse-height analysis. 


\section{WSRC-TR-2001-00584, Revision 0}

SRT-RPP-2001-00220, Revision 0

\section{C.2 Comparison of Melter-Scale "Regulatory" Analyses to Other Tank AN-102 Results}

Table 5.11 includes average results from this report, similar results determined at SRTC, the Washington Administrative Code radionuclide requirements for this waste form and results from characterization of a similar waste form that was produced on a crucible scale. Table 5.11 shows

-All results indicate the waste forms met requirements of the Washington Administrative Code. -Individual radionuclide results were similar for the BWXT and the SRTC results.

-Technetium-99 concentrations were higher in the crucible-scale waste form than melter-scale.

All results met the Washington Administrative Code (WAC) radionuclide requirements. The total alpha requirement given in Table 5.11 is actually a limit on the transuranic alpha-emitting radionuclides. Comparing the total alpha concentration to the requirement is conservative. If the total alpha content is less than the limit, the transuranic alpha will be. The cesium content of the waste was almost two order magnitudes less than the WAC limit. Strontium was almost an order of magnitude below the limit, and technetium was between one half and one fifth the limit.

Results from the BWXT and SRTC measurements were similar for the individual radionuclides for both the melter and crucible tests. In addition, for the crucible tests, BWXT and SRTC results were similar for the total beta and total alpha results. For the melter tests, the BWXT total beta results were about $70 \%$ of the SRTC values. The total activity of the measured betaemitting radionuclides seems to have been more in line with the BWXT results.

As shown in Table 5.11, the measured radionuclide content in the melter-scale waste form was similar to the radionuclide content in the crucible-scale waste form for all primary radionuclides except plutonium-241 and technetium-99. The discrepancy in the plutonium-241 values appeared to be due to error associated with measuring concentrations close to the MDA. Low values for the melter-scale technetium-99 concentrations were consistent with detection of technetium in the melter off gas. ${ }^{4}$

\section{D Volatile Organic Compounds}

As expected, no volatile organic compounds were determined to be in the glass samples. Acetone and tetrahydrofuran were detected in the samples, but were detected in the field and trip blanks at similar concentrations. THF was also detected in the method blank at a similar concentration. These results have been given in Table 5.12. Of the 81 analytes initially on the list of target volatile organic analytes, the 14 compounds given in Table 5.12 were the only species that were either shown to be present in the melter feed or which could not be determined in the melter feed. Percent completeness for this set of results was $88 \%$ (30 valid results of the 34 values). 
WSRC-TR-2001-00584, Revision 0

SRT-RPP-2001-00220, Revision 0

Table 5.11. Comparison of Target Radionuclides

\begin{tabular}{|c|c|c|c|c|c|}
\hline & \multicolumn{3}{|c|}{ Melter Waste Form (pCi/kg) } & \multicolumn{2}{|c|}{ Crucible Waste Form (pCi/kg) } \\
\hline & BWXT & SRTC & WAC & BWXT & SRTC \\
\hline Alpha & $2.1 \pm 0.37 \times 10^{7}$ & $9.5 \pm 0.10 \times 10^{6}$ & $<1 \times 10^{8}$ & $2.5 \pm 0.74 \times 10^{7}$ & $2.20 \pm 0.035 \times 10^{7}$ \\
\hline Beta & $1.7 \pm 0.11 \times 10^{9}$ & $2.6 \pm 0.31 \times 10^{9}$ & - & $1.94 \pm 0.056 \times 10^{9}$ & $3.1 \pm 0.11 \times 10^{9}$ \\
\hline Se-79 & $<6 \times 10^{6}$ & - & - & ${ }^{\mathrm{J}}<9 \times 10^{6}$ & - \\
\hline Np-237 & ${ }^{\mathrm{U}_{1}} .9 \pm 1.2 \times 10^{4}$ & - & - & $<1 \times 10^{4}$ & - \\
\hline Pu239/240 & $5.5 \pm 0.23 \times 10^{5}$ & $3.4 \pm 4.2 \times 10^{5}$ & - & $6.32 \pm 0.066 \times 10^{5}$ & $<3 \times 10^{5}$ \\
\hline $\mathrm{Pu}-238$ & $4.6 \pm 0.11 \times 10^{5}$ & $5.5 \pm 0.99 \times 10^{5}$ & - & $5.1 \pm 0.30 \times 10^{5}$ & $<6 \times 10^{5}$ \\
\hline $\mathrm{Pu}-241$ & $1.29 \pm 0.045 \times 10^{6}$ & $1.8 \times 10^{6}$ & - & $2.4 \times 10^{6}$ & $<3 \times 10^{6}$ \\
\hline Am-241 & $8.33 \pm 0.038 \times 10^{6}$ & $4.1 \pm 0.53 \times 10^{6}$ & - & $1.03 \pm 0.063 \times 10^{7}$ & $1.3 \pm 0.13 \times 10^{7}$ \\
\hline *Am-241 & $9.6 \pm 1.3 \times 10^{6}$ & - & - & - & - \\
\hline $\mathrm{Cm}-244$ & $5.79 \pm 0.068 \times 10^{6}$ & $2.3 \pm 0.34 \times 10^{6}$ & - & $8.1 \pm 0.59 \times 10^{6}$ & $8.2 \pm 1.1 \times 10^{6}$ \\
\hline $\mathrm{Cm}-242$ & $3.2 \pm 0.75 \times 10^{4}$ & - & - & $5.4 \times 10^{4}$ & - \\
\hline U-234 & $8.2 \times 10^{4}$ & - & - & $1.61 \pm 0.054 \times 10^{4}$ & - \\
\hline $\mathrm{U}-235$ & $\mathrm{~J}^{4} .3 \pm 2.9 \times 10^{3}$ & - & - & $<7 \times 10^{3}$ & - \\
\hline U-236 & $<9 \times 10^{2}$ & - & - & $<7 \times 10^{3}$ & - \\
\hline U-238 & $4.9 \pm 0.78 \times 10^{3}$ & - & - & $1.1 \times 10^{4}$ & - \\
\hline I-129 & ${ }^{\mathrm{U}} 1.2 \pm 0.10 \times 10^{6}$ & - & - & $<2 \times 10^{5}$ & - \\
\hline Tc-99 & $5.82 \pm 0.017 \times 10^{6}$ & $6.5 \pm 0.11 \times 10^{6}$ & $<3 \times 10^{7}$ & $1.67 \pm 0.040 \times 10^{7}$ & $1.7 \pm 0.13 \times 10^{7}$ \\
\hline Sr-90 & $8.2 \pm 0.98 \times 10^{8}$ & $7.0 \pm 1.5 \times 10^{8}$ & $<7 \times 10^{9}$ & $9.95 \pm 0.72 \times 10^{8}$ & $1.11 \pm 0.042 \times 10^{9}$ \\
\hline Co-60 & $2.0 \pm 0.10 \times 10^{7}$ & $2.09 \pm 0.021 \times 10^{7}$ & - & $2.73 \times 10^{7}$ & $3.3 \pm 0.15 \times 10^{7}$ \\
\hline $\mathrm{Nb}-94$ & $1.9 \times 10^{6}$ & - & - & $<1 \times 10^{5}$ & - \\
\hline Ru-103 & $<1 \times 10^{5}$ & - & - & $<1 \times 10^{5}$ & - \\
\hline Ru-106 & $<1 \times 10^{6}$ & - & - & $<1 \times 10^{6}$ & - \\
\hline Cs-134 & $<1 \times 10^{5}$ & - & - & $<1 \times 10^{5}$ & - \\
\hline Cs-137 & $2.3 \pm 0.11 \times 10^{7}$ & $2.55 \times 10^{7}$ & $<1 \times 10^{9}$ & $3.18 \pm 0.019 \times 10^{7}$ & $3.41 \pm 0.042 \times 10^{7}$ \\
\hline Eu-152 & $3.2 \times 10^{5}$ & 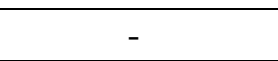 & - & $4.8 \times 10^{5}$ & - \\
\hline Eu-154 & $2.0 \pm 0.23 \times 10^{7}$ & $1.8 \pm 0.22 \times 10^{7}$ & - & $2.74 \pm 0.025 \times 10^{7}$ & - \\
\hline Eu-155 & $1.1 \pm 0.11 \times 10^{7}$ & $1.1 \pm 0.48 \times 10^{7}$ & - & $1.62 \pm 0.011 \times 10^{7}$ & - \\
\hline Ra-226 & $<2 \times 10^{5}$ & - & - & ${ }^{\mathrm{U}} 6.2 \times 10^{5}$ & - \\
\hline C-14 & ${ }^{R} 2.9 \times 10^{6}$ & - & - & $9.3 \pm 0.71 \times 10^{6}$ & - \\
\hline H-3 & $<2 \times 10^{6}$ & - & - & $<2 \times 10^{6}$ & - \\
\hline
\end{tabular}

"<"indicates a result that was less than the MDA. This is usually indicated by a "U" qualifier.

${ }^{\mathrm{J}}$ Analyte regarded as detected, but validation showed its reported concentration to be uncertain.

${ }^{\mathrm{R}}$ The analyte results was determined to be unusable during data validation.

UThe analyte was regarded as not detected during data validation.

*These americium-241 results were determined from the gamma pulse-height analysis. 
WSRC-TR-2001-00584, Revision 0

SRT-RPP-2001-00220, Revision 0

Table 5.12. Concentrations of Target Volatile Organic Analytes

\begin{tabular}{|c|c|c|c|c|c|c|}
\hline & \multicolumn{3}{|c|}{ Samples $(\mu \mathbf{g} / \mathbf{k g})$} & \multicolumn{3}{|c|}{ Process Blanks $(\mu \mathrm{g} / \mathbf{k g})$} \\
\hline & VA1 & VA2 & Average & VP1 & VP2 & Average \\
\hline *Chloromethane & $<10$ & $<10$ & $<10$ & $<10$ & $<10$ & $<10$ \\
\hline *Vinyl Chloride & $<10$ & $<10$ & $<10$ & $<10$ & $<10$ & $<10$ \\
\hline Acetone & J 8.9 & $\mathrm{~J}_{8.0}$ & ${ }_{8.5 \pm 0.64}$ & 10.4 & ${ }^{\mathrm{J}} 10.1$ & $10.3 \pm 0.21$ \\
\hline Methylene Chloride & $<10$ & $<10$ & $<10$ & $<10$ & $<10$ & $<10$ \\
\hline 2-Butanone & $<10$ & $<10$ & $<10$ & $<10$ & $<10$ & $<10$ \\
\hline 1,2-Dichloroethane & $<10$ & $<10$ & $<10$ & $<10$ & $<10$ & $<10$ \\
\hline 2-Butenal & $<10$ & $<10$ & $<10$ & $<10$ & $<10$ & $<10$ \\
\hline 2-Methyl-2-Propanol & $<1 \times 10^{2}$ & $<1 \times 10^{2}$ & $<1 \times 10^{2}$ & $<1 \times 10^{2}$ & $<1 \times 10^{2}$ & $<1 \times 10^{2}$ \\
\hline Ethylene Oxide & $<10$ & $<10$ & $<10$ & $<10$ & $<10$ & $<10$ \\
\hline Tetrahydrofuran & $\mathrm{JU}^{\mathrm{JU}} 6.2$ & ${ }^{\mathrm{J}} 6.5$ & ${ }^{\mathrm{JU}} 6.4 \pm 0.21$ & $\mathrm{JU}_{7.5}$ & ${ }^{\mathrm{JU}} 7.2$ & $\mathrm{JU}_{7.4 \pm 0.21}$ \\
\hline 2-Butanol & $<1 \times 10^{2}$ & $<1 \times 10^{2}$ & $<1 \times 10^{2}$ & $<1 \times 10^{2}$ & $<1 \times 10^{2}$ & $<1 \times 10^{2}$ \\
\hline Isopropanol & $<1 \times 10^{2}$ & $<1 \times 10^{2}$ & $<1 \times 10^{2}$ & $\mathrm{~J}_{2.8}$ & $<1 \times 10^{2}$ & $\mathrm{~J}_{2.8}$ \\
\hline & IA1 & IA2 & Average & IP1 & IP2 & Average \\
\hline **Methanol & $<2 \times 10^{4}$ & $<2 \times 10^{4}$ & $<2 \times 10^{4}$ & $<1 \times 10^{4}$ & $<1 \times 10^{4}$ & $<1 \times 10^{4}$ \\
\hline **2-Butoxyethanol & $<2 \times 10^{4}$ & $<2 \times 10^{4}$ & $<2 \times 10^{4}$ & $<1 \times 10^{4}$ & $<1 \times 10^{4}$ & $<1 \times 10^{4}$ \\
\hline Acetate & ${ }^{\mathrm{J}} 14.5$ & $<21$ & ${ }^{\mathrm{J}} 14.5$ & $<19$ & $<22$ & $<19$ \\
\hline Formate & $<15$ & $<21$ & 15 & $<19$ & $<22$ & $<19$ \\
\hline Oxalate & $<15$ & ${ }^{\mathrm{J}} 11.5$ & ${ }^{\mathrm{J}} 11.5$ & $<38$ & $<44$ & $<38$ \\
\hline
\end{tabular}

"<"indicates a result that was less than the EQL. This is usually indicated by a "U" qualifier.

${ }^{\mathrm{J}}$ Analyte was detected but at less than the estimated quantitation limit.

UAnalyte regarded as not detected during data validation (method blank concentration $8.4 \mu \mathrm{g} / \mathrm{kg}$ ).

*During validation, results were given a "J" data qualifier because laboratory control sample recoveries below $80 \%$.

**During validation, results were given a "J" data qualifier because analyses outside hold times.

\section{E Semivolatile Organic Compounds}

Results from the semivolatile organic compound determinations have been given in Table 5.13. Semivolatile organic compound analyses showed that

-No target semivolatile organic compounds were detected in the Tank AN-102 glass waste form. -Tentatively-identified compound results were similar for the samples and the blanks.

-Triethylamine results were rejected during data validation.

As expected, none of the target semivolatile organic compounds were detected in the Tank AN102 glass waste form. This is consistent with results from organic compound analyses performed on other Hanford-site glass waste forms and should be expected since the waste form was produced at $1150{ }^{\circ} \mathrm{C}$. At this temperature, all organic compounds were expected to have been decomposed or volatilized. 
WSRC-TR-2001-00584, Revision 0

SRT-RPP-2001-00220, Revision 0

Table 5.13. Concentrations of Target Semivolatile Organic Analytes

\begin{tabular}{|c|c|c|c|c|}
\hline & \multicolumn{3}{|c|}{ Samples $(\mu \mathbf{g} / \mathbf{k g})$} & Process Blanks $(\mu \mathrm{g} / \mathrm{kg})$ \\
\hline & SA1 & SA2 & Average & Average \\
\hline N-Nitrosodimethylamine & $<3 \times 10^{2}$ & $<7 \times 10^{2}$ & $<3 \times 10^{2}$ & $<3 \times 10^{2}$ \\
\hline Phenol & $<3 \times 10^{2}$ & $<7 \times 10^{2}$ & $<3 \times 10^{2}$ & $<3 \times 10^{2}$ \\
\hline 1,3,-Dichlorobenzene & $<3 \times 10^{2}$ & $<7 \times 10^{2}$ & $<3 \times 10^{2}$ & $<3 \times 10^{2}$ \\
\hline 1,4-Dichlorobenzene & $<3 \times 10^{2}$ & $<7 \times 10^{2}$ & $<3 \times 10^{2}$ & $<3 \times 10^{2}$ \\
\hline Benzyl Alcohol & $<3 \times 10^{2}$ & $<7 \times 10^{2}$ & $<3 \times 10^{2}$ & $<3 \times 10^{2}$ \\
\hline 1,2-Dichlorobenzene & $<3 \times 10^{2}$ & $<7 \times 10^{2}$ & $<3 \times 10^{2}$ & $<3 \times 10^{2}$ \\
\hline 2-Methylphenol & $<3 \times 10^{2}$ & $<7 \times 10^{2}$ & $<3 \times 10^{2}$ & $<3 \times 10^{2}$ \\
\hline 4-Methylphenol & $<3 \times 10^{2}$ & $<7 \times 10^{2}$ & $<3 \times 10^{2}$ & $<3 \times 10^{2}$ \\
\hline Hexachloroehtane & $<3 \times 10^{2}$ & $<7 \times 10^{2}$ & $<3 \times 10^{2}$ & $<3 \times 10^{2}$ \\
\hline Nitrobenzene & $<3 \times 10^{2}$ & $<7 \times 10^{2}$ & $<3 \times 10^{2}$ & $<3 \times 10^{2}$ \\
\hline Propyl Nitrate & $<3 \times 10^{2}$ & $<7 \times 10^{2}$ & $<3 \times 10^{2}$ & $<3 \times 10^{2}$ \\
\hline Acetophenone & $<3 \times 10^{2}$ & $<7 \times 10^{2}$ & $<3 \times 10^{2}$ & $<3 \times 10^{2}$ \\
\hline 1,2,4-Trichlorobenzene & $<3 \times 10^{2}$ & $<7 \times 10^{2}$ & $<3 \times 10^{2}$ & $<3 \times 10^{2}$ \\
\hline Naphthalene & $<3 \times 10^{2}$ & $<7 \times 10^{2}$ & $<3 \times 10^{2}$ & $<3 \times 10^{2}$ \\
\hline Hexachlorobutadiene & $<3 \times 10^{2}$ & $<7 \times 10^{2}$ & $<3 \times 10^{2}$ & $<3 \times 10^{2}$ \\
\hline 2,4-Dinitrotoluene & $<3 \times 10^{2}$ & $<7 \times 10^{2}$ & $<3 \times 10^{2}$ & $<3 \times 10^{2}$ \\
\hline 1,4-Dinitrobenzene & $<3 \times 10^{2}$ & $<7 \times 10^{2}$ & $<3 \times 10^{2}$ & $<3 \times 10^{2}$ \\
\hline Diphenylamine & $<3 \times 10^{2}$ & $<7 \times 10^{2}$ & $<3 \times 10^{2}$ & $<3 \times 10^{2}$ \\
\hline 1,1-Biphenyl & $<3 \times 10^{2}$ & $<7 \times 10^{2}$ & $<3 \times 10^{2}$ & $<3 \times 10^{2}$ \\
\hline BHT & $<3 \times 10^{2}$ & $<7 \times 10^{2}$ & $<3 \times 10^{2}$ & $<3 \times 10^{2}$ \\
\hline Tributyl Phosphate & $<3 \times 10^{2}$ & $<7 \times 10^{2}$ & $<3 \times 10^{2}$ & $<3 \times 10^{2}$ \\
\hline Pentachlorophenol & $<3 \times 10^{2}$ & $<7 \times 10^{2}$ & $<3 \times 10^{2}$ & $<3 \times 10^{2}$ \\
\hline PCNB & $<3 \times 10^{2}$ & $<7 \times 10^{2}$ & $<3 \times 10^{2}$ & $<3 \times 10^{2}$ \\
\hline Dinoseb & $<3 \times 10^{2}$ & $<7 \times 10^{2}$ & $<3 \times 10^{2}$ & $<3 \times 10^{2}$ \\
\hline Tetrachloronaphthalene & $<3 \times 10^{2}$ & $<7 \times 10^{2}$ & $<3 \times 10^{2}$ & $<3 \times 10^{2}$ \\
\hline bis (2-ethylhexyl) Phthalate & $<3 \times 10^{2}$ & $<7 \times 10^{2}$ & $<3 \times 10^{2}$ & $<3 \times 10^{2}$ \\
\hline Di-n-Octylphthalate & $<3 \times 10^{2}$ & $<7 \times 10^{2}$ & $<3 \times 10^{2}$ & $<3 \times 10^{2}$ \\
\hline Benzo (a) Pyrene & $<3 \times 10^{2}$ & $<7 \times 10^{2}$ & $<3 \times 10^{2}$ & $<3 \times 10^{2}$ \\
\hline Dibenzo (a,h) Anthracene & $<3 \times 10^{2}$ & $<7 \times 10^{2}$ & $<3 \times 10^{2}$ & $<3 \times 10^{2}$ \\
\hline p-Nitrochlorobenzene & $<3 \times 10^{2}$ & $<7 \times 10^{2}$ & $<3 \times 10^{2}$ & $<3 \times 10^{2}$ \\
\hline 2-Propenoic acid & $<3 \times 10^{2}$ & $<7 \times 10^{2}$ & $<3 \times 10^{2}$ & $<3 \times 10^{2}$ \\
\hline Triethylamine & $<3 \times 10^{2}$ & $<7 \times 10^{2}$ & $<3 \times 10^{2}$ & $<3 \times 10^{2}$ \\
\hline *Toluene & $8.1 \times 10^{3}$ & $1.6 \times 10^{4}$ & $1.2 \pm 0.56 \times 10^{4}$ & $8.0 \times 10^{3}$ \\
\hline *1,2-Benzenedicarboxylic Acid & $1.4 \times 10^{3}$ & $1.2 \times 10^{3}$ & $1.3 \pm 0.14 \times 10^{3}$ & $8.2 \pm 0.26 \times 10^{2}$ \\
\hline *N-Hexadecanoic Acid & $4.1 \times 10^{2}$ & $5.1 \times 10^{2}$ & $4.6 \pm 0.71 \times 10^{2}$ & $4.6 \pm 1.4 \times 10^{2}$ \\
\hline *Dibutyl Phthalate & $1.0 \times 10^{3}$ & $1.5 \times 10^{3}$ & $1.3 \pm 0.35 \times 10^{3}$ & $6.4 \pm 8.5 \times 10^{2}$ \\
\hline *Octadecanoic Acid & - & - & - & $1.9 \pm 0.64 \times 10^{2}$ \\
\hline
\end{tabular}




\section{WSRC-TR-2001-00584, Revision 0 \\ SRT-RPP-2001-00220, Revision 0}

*These were nontarget analytes determined as TICs. Results are estimates ("J" qualifier).

As shown in Table 5.13, five semivolatile compounds were detected in the samples. These compounds were also detected in the process blanks, field blanks and trip blanks at similar concentrations. Three of these compounds (toluene, 1,2-benzenedicarboxylic acid, and dibutyl phthalate) were also detected in the laboratory method blank at similar concentrations. By application of the CLP 5x rule, these compounds should be considered to be not detected in the samples.

In addition to the USEPA SW-846 Method 8270C determinations, triethylamine results were also given in Table 5.13. Triethylamine was determined by a separate ICP-MS method. Although the laboratory found no evidence for this target analyte, several quality control and quality assurance indicators were outside of specifications. As a result, the triethylamine results were rejected during data validation. Further development work will be needed before this method will meet the quality requirements of comparable USEPA SW-846 methods.

Oxalate results have been given previously (in Table 5.13) since it was determined along with formate and acetate by USEPA Method 9056. Although oxalate was detected in one of the samples, it was also detected in one of the field blanks and in the trip blank at higher concentrations. Therefore, by application of the CLP 5x rule, oxalate should be considered to be undetected in the samples.

\section{F Pesticides and Polychlorinated Biphenyls (PCBs)}

As expected, no pesticide or polychlorinated biphenyl (PCB) was detected in the Tank AN-102 glass waste form. This was consistent with results from organic compound analyses performed on other Hanford-site glass waste forms and should be expected since the waste form was produced at temperatures expected to have caused these species to be decomposed or volatilized. Results from the pesticide and polychlorinated biphenyl (PCB) analyses have been given in Table 5.14 .

\section{G Dioxins and Furans}

As expected, no dioxin or furan was found to be present in the Tank AN-102 glass waste form. Results from these analyses have been given in Table 5.15. These results indicated that although ten of these species were detected in one or both samples, these species were also detected in the extraction method blank at similar concentrations. Therefore, by application of the CLP 5x rule, these species should be considered to be undetected in the samples. The process blank results have been presented along with the sample results in Table 5.15. 
Table 5.14. Concentrations of Pesticides and Polychlorinated Biphenyls

\begin{tabular}{|c|c|c|c|c|}
\hline & \multicolumn{3}{|c|}{ Samples $(\mu \mathrm{g} / \mathbf{k g})$} & Process Blanks $(\mu \mathrm{g} / \mathbf{k g})$ \\
\hline & PA1 & PA2 & Average & Average \\
\hline Aldrin & $<3$ & $<3$ & $<3$ & $<2$ \\
\hline alpha-BHC & $<3$ & $<3$ & $<3$ & $<2$ \\
\hline beta-BHC & $<3$ & $<3$ & $<3$ & $<2$ \\
\hline gamma-BHC (Lindane) & $<3$ & $<3$ & $<3$ & $<2$ \\
\hline $\mathbf{4 , 4}$-DDD & $<7$ & $<6$ & $<6$ & $<3$ \\
\hline $\mathbf{4 , 4}$ 'DDT & $<7$ & $<6$ & $<6$ & $<3$ \\
\hline Dieldrin & $<7$ & $<6$ & $<6$ & $<3$ \\
\hline Endrin & $<7$ & $<6$ & $<6$ & $<3$ \\
\hline Heptachlor & $<3$ & $<3$ & $<3$ & $<2$ \\
\hline Toxaphene & $<3 \times 10^{2}$ & $<3 \times 10^{2}$ & $<3 \times 10^{2}$ & $<2 \times 10^{2}$ \\
\hline Isodrin & $<7$ & $<6$ & $<6$ & $<3$ \\
\hline Hexachlorobenzene & $<7$ & $<6$ & $<6$ & $<3$ \\
\hline Octachloronaphthalene & $<7$ & $<6$ & $<6$ & $<33$ \\
\hline${ }^{\mathbf{N}}$ Arochlor-1016 & $<67$ & $<64$ & $<64$ & $<67$ \\
\hline${ }^{\mathbf{N}}$ Arochlor-1221 & $<1 \times 10^{2}$ & $<1 \times 10^{2}$ & $<1 \times 10^{2}$ & $<33$ \\
\hline${ }^{\mathbf{N}}$ Arochlor-1232 & $<67$ & $<64$ & $<64$ & $<33$ \\
\hline${ }^{\mathbf{N}}$ Arochlor-1242 & $<67$ & $<64$ & $<64$ & $<33$ \\
\hline${ }^{\mathbf{N}}$ Arochlor-1248 & $<67$ & $<64$ & $<64$ & $<33$ \\
\hline${ }^{\mathbf{N}}$ Arochlor-1254 & $<67$ & $<64$ & $<64$ & $<33$ \\
\hline${ }^{\mathbf{N}}$ Arochlor-1260 & $<67$ & $<64$ & $<64$ & 464 \\
\hline
\end{tabular}

"<"indicates a result that was less than the EQL. This is usually indicated by a "U" qualifier.

${ }^{N}$ RPD for the two PCB spiked compounds did not meet the specification of less than $20 \%$.

\section{H Physical Properties}

As expected, the Tank AN-102 glass waste form was determined to be not flammable at temperatures above $1200{ }^{\circ} \mathrm{C}$. Density measurements were also performed; however, these results are not being reported. Due to limited sample availability, error associated with determining a small mass by difference of two larger masses caused the resulting density results to be unrealistic and to have poor precision. The field blank density measurement was similarly affected. In future analyses, more sample will be allocated for density measurements. 
WSRC-TR-2001-00584, Revision 0

SRT-RPP-2001-00220, Revision 0

Table 5.15. Concentrations of Dioxins and Furans

\begin{tabular}{|c|c|c|c|c|}
\hline & \multicolumn{3}{|c|}{ Samples (ng/kg) } & Process Blanks (ng/kg) \\
\hline & DA1 & DA2 & Average & Average \\
\hline 2378-TCDD & $<0.2$ & $<0.1$ & $<0.1$ & $<0.1$ \\
\hline 12378-PeCDD & $<0.2$ & $<0.1$ & $<0.1$ & J 0.47 \\
\hline 123478-HxCDD & $<0.2$ & $<0.1$ & $<0.1$ & $<0.1$ \\
\hline 123678-HxCDD & 0.25 & $<0.1$ & 0.25 & $0.57 \pm 0.56$ \\
\hline 123789-HxCDD & ${ }^{\mathrm{J}}(0.17)$ & $<0.1$ & $<0.1$ & 0.65 \\
\hline 1234678-HpCDD & ${ }^{\mathrm{J}}(0.44)$ & $\mathrm{J}^{\mathrm{J}} 0.44$ & J 0.44 & $0.76 \pm 0.63$ \\
\hline OCDD & $\mathrm{J}_{0.82}$ & 0.82 & ग 0.82 & ${ }^{\mathrm{J}} 1.2 \pm 0.52$ \\
\hline 2378-TCDF & J 0.66 & $<0.08$ & 0.66 & 0.47 \\
\hline 12378-PeCDF & J 0.19 & $<0.08$ & 0.19 & 0.36 \\
\hline 23478-PeCDF & 0.21 & $<0.08$ & 0.21 & 0.16 \\
\hline 123478-HxCDF & 0.23 & 0.1 & $\mathrm{~J} 0.165 \pm 0.092$ & 0.47 \\
\hline 123678-HxCDF & $\mathrm{J}_{0.15}$ & $<0.08$ & 0.15 & $0.16 \pm 0.099$ \\
\hline 234678-HxCDF & $<0.1$ & $<0.09$ & $<0.09$ & $<0.1$ \\
\hline 123789-HxCDF & $<0.2$ & $<0.1$ & $<0.1$ & $<0.1$ \\
\hline 1234678-HpCDF & ${ }^{\mathrm{J}}(0.29)$ & $<0.1$ & $<0.1$ & $<0.1$ \\
\hline 1234789-HpCDF & $<0.2$ & $<0.1$ & $<0.1$ & $<0.1$ \\
\hline OCDF & $<0.3$ & $<0.2$ & $<0.2$ & $<0.2$ \\
\hline Total TCDD & 0.35 & $(0.19)$ & 0.35 & $0.41 \pm 0.15$ \\
\hline Total PeCDD & $(1.0)$ & $(0.32)$ & $(0.32)$ & $1.9 \pm 1.7$ \\
\hline Total HxCDD & 0.63 & $(0.57)$ & 0.63 & $1.8 \pm 1.5$ \\
\hline Total HpCDD & 0.3 & 0.3 & 0.3 & $1.2 \pm 1.3$ \\
\hline Total TCDF & 2.3 & $<0.08$ & 2.3 & $1.4 \pm 0.57$ \\
\hline Total PeCDF & 0.4 & $<0.08$ & 0.4 & $1.1 \pm 1.4$ \\
\hline Total HxCDF & 0.38 & 0.1 & $0.24 \pm 0.20$ & $0.68 \pm 0.60$ \\
\hline Total HpCDF & $(0.29)$ & $<0.1$ & $<0.1$ & $<0.1$ \\
\hline
\end{tabular}

"<"indicates a result that was less than the EQL. This is usually indicated by a "U" qualifier.

${ }^{\mathrm{J}}$ Concentration was below the calibration curve, and analyte was detected in the method blank.

( ) Parentheses indicated a value that is the estimated maximum possible concentration.

\subsection{Conclusion/Summary}

These analyses were successful at determining the composition of the glass waste form composition. As a result, the waste form was shown to meet requirements of the Washington Administrative Code. Comparing the results from these Tank AN-102 glass waste form samples (produced in a bench-scale melter) to results from a smaller study of this waste form (cruciblescale) showed the only large difference was in the technetium-99 concentrations. 


\section{WSRC-TR-2001-00584, Revision 0 \\ SRT-RPP-2001-00220, Revision 0}

\section{A Metals}

Elemental analyses were successful at determining the composition of the Tank AN-102 glass waste form. These analyses showed

-The glass waste form elemental composition was similar to the target composition.

-Analyses meet the reporting requirements of the WAC after accounting for titanium.

-The waste form met UTS requirements for all metals that were determined in the TCLP extract.

-Results from analyses performed by BWXT were similar to results from SRTC analyses.

-The melter-scale waste form composition was similar to the crucible-scale glass composition.

Elemental analysis of the dissolved glass samples showed that the composition of the melter glass waste form was similar to the target composition that had been developed by the Vitreous State Laboratory at Catholic University. All species detected above the estimated quantitation levels (EQLs) were within $20 \%$ of the target concentrations.

On an oxide basis, the total weight percent accounted for in these analyses was $100 \%$ after including titanium which was not a target analyte for these measurements. In future studies, titanium will be included in the list of target analytes along with any other element (except oxygen) that is in the glass forming chemicals at concentrations greater than $0.1 \%$.

Results from analysis of the TCLP extract showed that the waste form met the universal treatment standard (UTS) requirements for all elements that were monitored. Vanadium and zinc were not determined in the extract. In addition, detection limits for these elements were too high to determine whether sufficient concentrations were available to pass TCLP under condition of complete waste form dissolution. In future studies, attempts will be made to either measure these analytes in the TCLP extract or lower the MDL in the dissolved glass samples.

Comparison of results from these studies to those from analyses performed at SRTC indicated that for all primary elements, the values were very similar for the two sets of results. Of the elements detected at concentrations above the EQL, only the barium concentrations varied by more than $10 \%$. The cause of this difference in the barium concentration has not yet been determined.

Comparison of results from the analyses of this melter glass waste form to results from the Tank AN-102 crucible-scale glass waste form showed the concentrations to be within $20 \%$ for all elements detected at concentrations above the EQL in both waste forms. Surprisingly, the values for one of the primary components, sodium, deviated by about $20 \%$. The SRTC values appear to have been more in line with the target concentrations.

In future waste form characterization tests, USEPA SW-846 Method 7610 will be used to determine potassium. This will lower the detection limit for this analyte. In addition, sodium will be determined by USEPA SW-846 Method 7770. Although the sodium values were adequate in these studies, the difference between the crucible and melter waste form 


\section{WSRC-TR-2001-00584, Revision 0 \\ SRT-RPP-2001-00220, Revision 0}

concentrations was larger than expected. Attempts will also be made in the next study to determine vanadium and zinc in the TCLP extract.

\section{B Inorganic Analytes Measured as Ionic Species}

Of the target inorganic analytes measured as ionic species, none were detected above the EQLs. Sulfate and total halide concentrations were above the MDLs. The total halide concentration was less than the target composition suggesting that most of the fluoride and chloride were volatilized during the vitrification. In the future, Method 9056 should be performed with and without the sodium removal steps to improve phosphate and sulfate detection limits.

\section{C Radionuclides}

Primary radionuclide concentrations were determined for this waste form. Results showed

-The waste form met the WAC specifications for radionuclide content.

-The total beta radioactivity in the glass waste form was $1.7 \times 10^{9} \mathrm{pCi} / \mathrm{kg}$.

-The total alpha radioactivity in the glass waste form was $2 \times 10^{7} \mathrm{pCi} / \mathrm{kg}$.

-Crucible-scale and melter-scale radionuclide results were similar except for technetium-99.

The WAC specifies a strontium-90 limit equivalent to $7 \times 10^{9} \mathrm{pCi} / \mathrm{kg}$ assuming a density of 2.87 $\mathrm{g} / \mathrm{cm}^{3}$. Although strontium-90 and its daughter radionuclide, yttrium-90, accounted for over 90 $\%$ of the activity in the waste form, it was still a factor of seven below this limit. Technetium-99 was a factor of 5 below the WAC limit, and cesium-137 was below the limit by a factor of forty. Although technetium-99 appears to have been lost during vitrification in the crucible-scale waste form, the loss seems to have been significantly higher in the melter-scale waste form.

Modifications are being made to the carbon-14 determination method to minimize the effect of interferences.

\section{D Organic Compounds}

As expected, no organic compound was found to be present in the glass waste form. This was consistent with results from analyses of crucible-scale glass waste forms from Tank AN-102 and from Tank AZ-102, and is consistent with the temperatures at which the waste forms have been prepared. In the few cases in which an organic was detected in the samples, similar or higher concentrations were detected in the associated method, trip and field blanks.

The triethylamine method will require further development for the technique to meet quality control requirements implemented in the USEPA SW-846 ICP-MS methods. In addition, acetate and formate cannot be separated using USEPA SW-846 Method 9056. Modifying the buffer used to elute the ion chromatography column could allow these analytes to be separated. Since RPP personnel have indicated that reporting the estimated maximum possible concentration is acceptable for these analytes, no plans are being made to implement this modification. 


\section{E Physical Properties}

As expected, the Tank AN-102 glass waste form was not flammable at temperatures up to 1200 ${ }^{\circ} \mathrm{C}$. Density measurements of these samples were hindered by use of a small sample. In future studies, larger samples will be allocated for use in the density determination. This will minimize the error associated with measuring a small sample mass using a difference of two significantly larger masses. 